\title{
Prekäre Balance: Überlegungen zum heiklen Verhältnis von Richtern und Gutachtern
}

\author{
Von Volker Boehme-Neßler, Berlin"
}

A. Macht der Gerichtsgutachter?

B. Unverzichtbarkeit - Gutachter und

Komplexität.

I. Komplexität der Welt ............. 191

II. Justiz in der Wissensgesellschaft..... 192

C. Richter und Gutachter - Klare Rollenverteilung in der Theorie?

I. Verfassungsrecht: Klare Rollenverteilung zwischen Richter und Gutachter....

II. Erkenntnistheorie: Fließende Grenzen statt klarer Rollen...

1. Sachfragen vs. Rechtsfragen scharfe Trennung? ............... 195

2. Normative Tätigkeit von Gutachtern

III. Widersprüchliches Recht - Unklare

Rollenverteilung im Gerichtssaal.... 197

1. Gesetzestechnik: Fachregel als Rechtsregel

2. Rechtsprechung: Bindung an das Gutachten als Regel ....

IV. Fazit: Unscharfe Rollenverteilung zwischen Gericht und Gutachter.... 200

D. Richter und Sachverständige - Sozialpsychologische Aspekte einer komplexen Beziehung. ...

I. Die Macht der Situation ............. 201

1. Sozialpsychologie: Situative Einflüsse auf menschliches Verhalten ........................... 201

2. Informationaler sozialer Einfluss .......................... 202

3. Autorität der Experten und des Gerichts

4. Fazit: Bewusstsein und Respekt.

II. Verzerrende Urteilsheuristiken ..... 203

1. Unverzichtbar - (Urteils)Heuristiken als Instrumente des Denkens ........................... 204

2. Verfügbarkeits- und Repräsentativitätsheuristik im Gerichtssaal ............................. 205 a) Verfügbarkeitsheuristik...... 205 b) Repräsentativitätsheuristik ....................... 206

c) Alltagstheorien in der Gerichtspraxis ............. 206

3. Der Ankereffekt im Gerichtssaal.......................... 207

4. Heuristiken und Gerichtsgutachten .......................... 208

a) Alltagstheorien und Gerichtsgutachten .................. 209

b) Ankereffekt und Gerichtsgutachten.................... 209

c) Fazit: Gerichtsgutachten als Chance und Risiko.......... 210

III. Die Rolle der Emotionen............. 210

1. Emotionen und Entscheidungen .......................... 210

2. Emotionen und Entscheidungen von Richtern ................... 211

3. Emotionen und Sachverständigengutachten ................. 211

IV. Die Suche nach Bestätigung - Der confirmation bias im Gerichtssaal ... 212

1. Der confirmation bias........... 212

2. Confirmation bias und Gerichtsgutachten..................... 213

E. Der Sachverständige als Gehilfe des Gerichts ........................... 213

I. Engel der Medizin - oder Reserveengel der Jurisprudenz? .............. 214

1. Überzeugungen, Werte, Vorurteile des Gutachters .............. 214

2. Ökonomische Abhängigkeiten. 215

II. Erwartungshaltung des Gerichts.... 215

III. Rollenbewusstsein und persönliche

Souveränität....................... 216

F. Der Richter und sein Berater.......... 217

I. Psychologische Grundlage: Selbstre-

flexion und Bewusstsein statt

Abwehr......................... 217

II. Ausserrechtliche Fachkompetenz :

Sachverständige auf der Richter-

bank?............................ 217

1. Kompetenz durch Gerichtsorganisation ....................... 217

* Prof. Dr. Dr. Volker Boehme-Neßler lehrt u.a. Öffentliches Recht, Rechtstheorie und Rechtssoziologie an der Hochschule für Technik und Wirtschaft Berlin. 
2. Fachkompetenz durch zertifizierte Fachrichter...

III. Doppelte Weichenstellung: Auswahl und Auftrag der Gutachter.......... 219

1. Das Gutachten.................. 219

2. Der Gutachter ................... 220

3. Das Wettbewerbsmodell........ 220

4. Der Gutachtenauftrag ........... 221

IV. Interdiszipinäre Kommunikations-

kompetenz ....

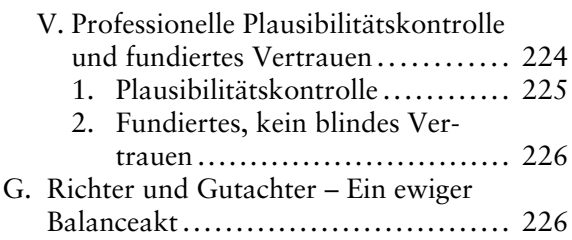

\section{A. Macht der Gerichtsgutachter?}

Das moderne Recht ist ohne Gutachter nicht denkbar. ${ }^{1}$ Das ist keine neue Erkenntnis. Untersuchungen zeigen, dass der faktische Einfluss von Gutachtern auf Urteile und Entscheidungen von Gerichten groß ist. ${ }^{2}$

In der Regel übernehmen Gerichte die Ergebnisse der Gutachter. ${ }^{3}$ Das gilt für alle Bereiche der Rechtsprechung. ${ }^{4}$ Die Grundlage für diesen Befund ist vielfältig: Empirische Untersuchungen einzelner Sachgebiete, Erfahrungsberichte von erfahrenen Richtern und Gutachtern über lange Zeiträume und zahlreiche Revisionsentscheidungen von Bundesgerichten, die eine unreflektierte Übernahme von Gutachtenergebnissen durch die Instanzgerichte bemängeln. ${ }^{5}$ Aus diesem Befund lässt sich ein Schluss ziehen, der auch tatsächlich oft gezogen wird: Die Macht der Gutachter ist zu groß. ${ }^{6}$ Der Gutachter soll ein unabhängiger, objektiver, fachkundiger Berater des Gerichts sein. ${ }^{7}$ Er darf das Gericht allerdings nicht durch seine Sachkunde dominieren.

1 Ähnlich M. Rehbinder, Rechtssoziologie, 7. Aufl., München 2009, Rn. 162; K. Detter, Der Sachverständige im Strafverfahren - eine Bestandsaufnahme, NStZ 1998, S. 57.

2 H. Kury/J. Obergfell-Fuchs, Rechtspsychologie, Stuttgart 2012, S. 111; W. Sass, Der Sachverständige weiterhin ein prozessuales Problemfeld, DS 2007, S. 256 (256 f. m.w.N.); I. Rode/A. Legnaro, Psychiatrische Sachverständige im Strafverfahren, München 1994, S. 24 f. auf der Basis empirischer Untersuchungen.

3 H. Kury/J. Obergfell-Fuchs (Fn. 3), S. 105; K. Foerster, in: FS H.-L. Schreiber, Heidelberg 2003, S. 81 (82 m. N. empirischer Studien); H. Franzki, Der Sachverständige - Diener oder Herr des Richters, DRiZ 1991, S. 314 (316); C.O. Schmidt/O.B. Scholz, Schuldfähigkeitsbegutachtung bei Tötungsdelikten, MSchrKrim 2000, S. 414 (423); W. Hörner u.a., Die Berücksichtigung des forensisch-psychiatrischen Gutachtens im Strafverfahren und im rechtskräftigen Urteil, MSchrKrim 1988, S. 395, (398 f.); S. Barton, Sachverständiger und Verteidiger, StV 1983, S. 73 (75 m.w.N.).

4 So zum Strafprozess K. Foerster, FS Schreiber (Fn. 4), S. 81 (82 f.); zum sozialgerichtlichen Verfahren K. Foerster, FS Schreiber (Fn. 4), S. 81 (83 f.); zum Arzthaftungsprozess C. Katzenmeier, in: A. Laufs/C. Katzenmeier/V. Lipp, Arztrecht, 6. Aufl. München 2009, Kap. XII. A. Rn. 7 m.w.N.; zum Zivilprozess H. Pieper u.a., Sachverständige im Zivilprozeß, München 1982, S. $261 \mathrm{ff}$. auf der Grundlage einer empirischen Untersuchung.

5 Etwa BGH NJW - RR 1995, 914.

6 H. Kury/J. Obergfell-Fuchs (Fn. 3), S. 105; K. Foerster, FS Schreiber (Fn. 4), S. 81 (82 ff. m. N. empirischer Studien); H. Franzki, Diener (Fn. 4), S. 314 (316); W. Hörner u.a., Berücksichtigung (Fn. 4), S. 395 (398f.).

7 BGHSt 9, 293 bezeichnet den Sachverständigen noch als „Gehilfen“ des Gerichts. Das ist aber kaum eine realitätsgerechte Charakterisierung. 
Aber ist das die zutreffende Deutung? Die Beziehung zwischen Gericht und Gutachter ist hoch komplex, von vielen Strukturen und Faktoren determiniert und beeinflusst. Dass Gerichte meistens den Gutachtern folgen, muss nicht das Ergebnis eines dominanten Diktats der Gutachter sein. Es kann auch die Folge eines gemeinsamen, anspruchsvollen und komplizierten Willensbildungsprozesses sein.

Zweifel an der einseitigen Dominanz-Hypothese wecken auch neuere empirische Untersuchungen. ${ }^{8}$ Danach werden Sachverständige im Kern ihrer Gutachtertätigkeit von Gerichten (zu) sehr beeinflusst. Gutachter spüren Erwartungen des Gerichts und erliegen der Versuchung, diese Erwartungen zu erfüllen. ${ }^{9}$ Dann verletzen sie die Rolle, die ihnen die Prozessordnungen zuweisen. Keinem Gutachter ist es erlaubt, sich vom Richter zu einem Gutachten drängen zu lassen, das Erwartungen des Gerichts lediglich bestätigt. ${ }^{10}$

Beide Beobachtungen scheinen sich zu widersprechen. Das ist ein Indiz dafür, dass sich die Beziehung zwischen Gericht und Gutachter nicht einfach holzschnittartig erklären lässt. Auch die These, dass eine Seite die andere dominiert, ist bei genauerem Hinsehen kaum haltbar. Es spricht einiges dafür, dass Richter und Sachverständige eine sehr komplexe, wechselseitige Beziehung auf Augenhöhe pflegen, in der beide Seiten ihre spezifischen Stärken haben. ${ }^{11}$

\section{B. Unverzichtbarkeit - Gutachter und Komplexität}

Die moderne Welt ist hoch technisierte und komplex. Kein Gericht kann ohne spezielle Fachgutachter alle unterschiedlichen Bereiche des Alltags verstehen. Gleichzeitig wachsen in der Wissensgesellschaft die Ansprüche an die Rationalität von Gerichtsentscheidungen. Insgesamt bedeutet das: Gutachter sind unverzichtbar - und sie werden es immer stärker.

\section{Komplexität der Welt}

Gerichte beschäftigen sich letztlich mit der Welt. Sie wenden rechtliche Regelungen auf Ereignisse aus allen Bereichen der Lebenswelt an. Richter ${ }^{12}$ kennen sich aber nur mit einem begrenzten Teil der Welt aus: dem Recht. Ohne objektive Fachleute - also Gutachter - könnten Richter deshalb einen großen Teil der Sachverhalte, die sie

8 B. Jordan/U. Gresser, Wie unabhängig sind Gutachter? DS 2014, S. 71.

9 Ausführlich dazu unten F.

10 Selbstverständlich ist es auch dem Richter nicht erlaubt, den Gutachter in eine bestimmte Richtung zu drängen.

11 Diese These müsste in einer umfangreichen Untersuchung empirisch bewiesen werden. Das kann hier nicht geleistet werden. In diesem Beitrag sollen theoretische Überlegungen angestellt werden, die diese These stützen.

12 Ein sehr hoher Prozentsatz der Richter sind Richterinnen. Trotzdem wird im Text nur von Richtern gesprochen. Das soll den Text vereinfachen und eine Konzentration auf das fokussierte Problem erleichtern. 
rechtlich würdigen sollen, nicht verstehen. Ein Teil der Welt lässt sich natürlich mit durchschnittlicher Allgemeinbildung und gesundem Menschenverstand erfassen und beurteilen. Die Welt wird allerdings in immer höherem Tempo und größerem Ausmaß von hochkomplexen Technologien geprägt. Beispiele dafür sind etwa die Gentechnik, die Nanotechnologie oder die digitale Technologie. Das hat eine brisante Folge für das Recht: Der Bereich der Welt, der ohne Gutachter verständlich ist, wird kleiner. Die Prozesse, mit denen sich die Gerichte beschäftigen müssen, werden ebenfalls komplexer und erfordern immer mehr hochspezifisches Fachwissen. Ohne Gutachter lassen sie sich immer weniger adäquat bewältigen.

\section{Justiz in der Wissensgesellschaft}

Moderne Industriegesellschaften sind Wissensgesellschaften. ${ }^{13}$ (Wissenschaftliches) Wissen ist die wichtigste gesellschaftliche Ressource für Weiterentwicklung und Wertschöpfung. Rationales Denken und Tag für Tag explosionsartig anwachsende Wissensmengen prägen zunehmend die Gesellschaft. ${ }^{14}$

Diese Entwicklung hat Folgen für das Recht. Das Rechtssystem in einer Wissensgesellschaft kann sich nicht mehr pragmatisch vor allem auf den „gesunden Menschenverstand" berufen. Es muss sich wissenschaftlich über die sozialen Sachverhalte informieren, die es regeln will. ${ }^{15}$ Und noch mehr: Es muss wissenschaftliche Erkenntnisse rezipieren und in seine Regelungen integrieren. Wissenschaftlich fundiertes Recht soll besser akzeptiert und legitimiert sein. ${ }^{16}$ Gleichzeitig erhofft man sich dadurch ein effektiveres Recht, das Verhalten und Entwicklungen in der modernen Industriegesellschaft zielgenauer steuern kann. ${ }^{17}$

Diese Tendenz betrifft alle Bereiche des Rechtssystems - natürlich auch die Justiz. Die Ansprüche an die wissenschaftliche Fundierung von Gerichtsentscheidungen steigen. Das zeigt exemplarisch die Diskussion um die freie Beweiswürdigung in $\$ 261$ StPO. Die freie Beweiswürdigung ist grundsätzlich sehr persönlich, vom Gefühl und der Intuition des Richters geprägt. ${ }^{18}$ Das passt wenig in das gesellschaftliche Umfeld, das immer stärker von wissenschaftlicher Rationalität beeinflusst wird.

13 Der Begriff der Wissensgesellschaft geht zurück auf $R$. Lane, The Decline of Politics and Ideology in a Knowledgeable Society, American Sociological Review, 1966 (31), S. 649; dazu ausführlich M. Heidenreich, Die Debatte um die Wissensgesellschaft, in: S. Böschen/I. Schulz-Schaeffer (Hrsg.): Wissenschaft in der Wissensgesellschaft, Wiesbaden 2003, S. 25 (25 ff. m.w.N.), der, a.a.O., S. 38, einen Überblick über die unterschiedlichen Vorstellungen von der Wissensgesellschaft gibt.

14 Grundsätzlich dazu D. Bell, The Coming of the Post-Industrial Society, New York 1985, S. 219 und pass.

15 M. Rehbinder, Rechtssoziologie (Fn. 2), Rn. 92.

16 M. Rebbinder, Rechtssoziologie (Fn. 2), Rn. 93.

17 M. Rebbinder, Rechtssoziologie (Fn. 2), Rn. 94.

18 R. Keller, Verwissenschaftlichung versus Rationalität der strafprozessualen Beweiswürdigung? GA 1999, S. 255. Selbstverständlich hat die Freiheit des Richters dabei auch Grenzen. Sie darf nicht zur Willkür verkommen. Immer noch grundlegend dazu BGHSt 10, 209 (211). Im einzelnen C. Roxin/B. Schünemann, Strafverfahrensrecht, 26. Aufl., München 2009, Rn. 50 f. m.w.N. 
Deshalb wird gefordert, die Beweiswürdigung stärker zu rationalisieren oder - zugespitzt - zu ,verwissenschaftlichen “. ${ }^{19}$ Der BGH hat sich weitgehend auf diese Forderungen eingelassen..$^{20}$ Die Folge: Immer öfter werden Gutachter beauftragt, um diese gestiegenen Ansprüche an die wissenschaftliche Rationalität von Gerichtsentscheidungen zu erfüllen. Richter alleine können diese Bedürfnisse nicht mehr erfüllen. ${ }^{21}$

Immer mehr Fragen können vor Gericht nur durch wissenschaftliche Gutachten beantwortet werden. Gleichzeitig sind die inhaltlich-fachlichen Ansprüche gestiegen, wenn es um Probleme geht, die vor Jahren noch ohne Sachverständige pragmatisch und adäquat gelöst worden wären. ${ }^{22}$

Die Entwicklung der Gesellschaft zur komplexen High-Tech-Gesellschaft geht weiter und vertieft sich. Das hat auch Auswirkungen auf das Rechtssystem im Allgemeinen und die Justiz im Besonderen. Die strukturelle Bedeutung der Sachverständigen im Rechtssystem nimmt weiter zu. Abgesehen von ganz einfachen Routineprozessen werden immer mehr Gerichtsverfahren unter Beteiligung von Sachverständigen durchgeführt. Das gilt - ohne Anspruch auf Vollständigkeit - für Zivilprozesse, Verwaltungsprozesse, sozialrechtliche Streitigkeiten genauso wie für familienrechtliche Auseinandersetzungen oder Strafprozesse. ${ }^{23}$

\section{Richter und Gutachter - Klare Rollenverteilung in der Theorie?}

\section{Verfassungsrecht: Klare Rollenverteilung zwischen Richter und Gutachter}

In der Demokratie geht - das ist der Grundsatz der Volkssouveränität - alle Staatsgewalt vom Volk aus. Rechtsprechung ist in der Demokratie also Aufgabe demokratisch legitimierter Gerichte. Das formuliert Art. 92 GG kurz und prägnant: Die rechtsprechende Gewalt ist den Richtern anvertraut. Und zwar den unabhängigen Richtern.

19 G. Fezer, Tatrichterlicher Erkenntnisprozeß - „Freiheit der Beweiswürdigung“ StV 1995, S. 95 (100); kritisch dazu aber R. Keller, Verwissenschaftlichung (Fn. 19), S. 255 (257 ff. m.w.N.).

20 So R. Keller, Verwissenschaftlichung (Fn. 19), S. 255, m.N. der Rspr. Allerdings lässt sich inzwischen - wie Keller (op.cit.) S. zeigt - ein Abrücken des BGH von der strikten Rationalitätsforderung beobachten.

21 Möglicherweise ist diese Entwicklung aber nicht unumkehrbar. Inzwischen rücken die Grenzen der Verwissenschaftlichung in den Fokus der Diskussion. Gleichzeitig lässt sich eine neue Wertschätzung von Erfahrungswissen feststellen. Ausführlich dazu F. Böhle, Wissenschaft und Erfahrungswissen Erscheinungsformen, Voraussetzungen und Folgen einer Pluralisierung des Wissens, in: S. Böschen/I. Schulz-Schaeffer (Hrsg.): Wissenschaft in der Wissensgesellschaft, Wiesbaden 2003, S. 143 (158ff. m.w.N.). Das könnte die nicht ausschließlich rationale richterliche Beweiswürdigung nach $\$ 261$ StPO wieder rehabilitieren.

22 Ein Beispiel sind die Familiengerichte, deren Fallgestaltungen relativ unabhängig vom technischen Fortschritt sind. Trotzdem nehmen auch dort die Gutachterbestellungen kontinuierlich und deutlich zu. Empirisches Material dazu bei U. Jopt/J. Zütphen, Psychologische Begutachtung aus familiengerichtlicher Sicht, ZfJ 2004 (91), S. 310 (314, 317).

23 Schätzungen dazu bei H. Kater, Das ärztliche Gutachten im sozialgerichtlichen Verfahren. 2. Aufl. Berlin 2011, S. 17 f. m.w.N. 
Dass Richter unabhängig sein sollen, ist ein Ideal, das vom Rechtsstaat gefordert wird. Art. 97 der Verfassung ist an Klarheit kaum zu überbieten: „Die Richter sind unabhängig und nur den Gesetzen unterworfen. “24 Im Rechtsstaat sind Richter, die nicht unabhängig sind, nicht denkbar. Das zeigt sich am Begriff der Rechtsprechung, wie ihn das Grundgesetz benutzt: Es gehört zum Wesen richterlicher Tätigkeit, dass sie unabhängig ausgeübt wird. ${ }^{25}$ Mit anderen Worten: Ohne Unabhängigkeit keine Rechtsprechung und keine Richter. ${ }^{26}$

Das hat tiefere Gründe: Nur unabhängige Richter können die Herrschaft des Rechts durchsetzen und Machtmissbrauch verhindern. Nur unabhängige Richter können den Drohungen, Manipulationen oder Verlockungen widerstehen, mit denen sie konfrontiert werden. Die Unabhängigkeit schafft erst den Freiraum, den Richter brauchen, um ihre Aufgabe im Rechtsstaat erfüllen zu können. ${ }^{27}$

Aber richterliche Unabhängigkeit ist nicht nur eine Frage der verfassungsrechtlichen Garantie. Mindestens ebenso wichtig ist die persönliche Souveränität, die im Alltag immer wieder auf die Probe gestellt wird. Ein rechtlich garantierter Freiraum nützt nichts, wenn ein Richter ihn nicht ausfüllt. Um es klar zu sagen: Wenn das Grundgesetz die rechtliche Unabhängigkeit der Gerichte garantiert, heißt das nicht zwingend, dass alle Richter in der Wirklichkeit des Gerichtssaals auch tatsächlich unabhängig sind. Notwendig ist die innere Unabhängigkeit und Souveränität des Richters, die er immer wieder selbst erarbeiten und verteidigen muss. ${ }^{28}$

Rechtsprechung erschöpft sich nicht in der formelhaften, formellen Verkündung von Urteilen. Sie ist ein materieller Erkenntnisprozess, an dessen Ende eine von Rechtsnormen vorstrukturierte Entscheidung mit verbindlicher Wirkung steht. Diese Entscheidung am Ende muss von dem dazu berufenen und durch die Berufung demokratisch legitimierten Richter getroffen werden. Basis der Entscheidung sind die Informationen, die im Verlauf eines rechtlich streng strukturierten Verfahrens gesammelt werden. Bei der Sammlung der Informationen spielen die Gutachter eine wich-

24 Ausführlich zum Gewährleistungsbereich von Art. 97 GG H.-J. Papier, Die richterliche Unabhängigkeit und ihre Schranken, NJW 2001, S. 1089 (1090 f. m.w.N.). Zur sachlichen und persönlichen Unabhängigkeit grundsätzlich BVerfGE 12, 81 (88 ff).; 26, 186 (198); 87, 68 (85).

25 BVerfGE 103, 111 (140).

26 Ähnlich C. Hillgruber, in T. Maunz/G. Dürig (Begr.), Grundgesetz Kommentar München 2014, Art. 97, Rn. 1: „Die in Art. 97 GG garantierte richterliche Unabhängigkeit ist für die dritte Gewalt schlechthin konstitutiv.".

27 C. Hillgruber (Fn. 27), Art. 97 Rn. 4 m.w.N.

28 H.-J. Papier, Die richterliche Unabhängigkeit und ihre Schranken, NJW 2001, S. 1089 (1091). Die innere Unabhängigkeit ist eine Haltung, die auch - nicht völlig - eingeübt und gelernt werden kann. Ähnlich H.-J. Faller, Die richterliche Unabhängigkeit im Spannungsfeld von Politik, Weltanschauung und öffentlicher Meinung, in: FS W. Zeidler, Berlin, New York 1987, S. 81 (98), der aber auch, a.a.O., S. 97 ff. weitere Faktoren herausarbeitet, die für die innere Unabhängigkeit der Richter relevant sind. 
tige Rolle. Sie sind letztlich wichtige Berater des Gerichts. ${ }^{29}$ Entscheider dürfen sie schon aus demokratietheoretischen Gründen nicht sein. ${ }^{30}$ Denn die Staatsgewalt Rechtsprechung wird in der Demokratie nur von demokratisch legitimierten Richtern ausgeübt. Die Entscheidung, welche rechtlichen Folgerungen aus dem im Prozess erarbeiteten Informationsmaterial zu ziehen sind, treffen im demokratischen Rechtsstaat exklusiv die Richter. ${ }^{31}$

\section{Erkenntnistheorie: Fließende Grenzen statt klarer Rollen}

Auf den ersten Blick ist die Rollenverteilung zwischen Gutachter und Richter also klar. Bei näherem Hinsehen wird die Grenzziehung zwischen Informationsbeschaffung auf der einen Seite und rechtlicher Bewertung und Entscheidung auf der anderen Seite aber schwierig und unscharf - möglicherweise sogar unmöglich. ${ }^{32}$

\section{Sachfragen vs. Rechtsfragen - scharfe Trennung?}

Auf den ersten Blick bewegen sich Sachverständige ${ }^{33}$ einerseits und Juristen andererseits in unterschiedlichen Denkwelten. Den - naturwissenschaftlichen, ingenieurwissenschaftlichen und medizinischen - Gutachtern geht es - so scheint es - um die kognitiv-rationale Erforschung der reinen Tatsachen. Richter dagegen arbeiten normativ und gestalten das Recht. ${ }^{34}$ Die Aufgabenverteilung im Prozess ist also scheinbar klar: Die fachlichen Sachfragen werden von den Sachverständigen bearbeitet. Die juristischen Bewertungsfragen dagegen sind Aufgabe des Gerichts. ${ }^{35}$

In Wirklichkeit lassen sich Sachfragen und Rechtsfragen nicht scharf trennen. Einiges spricht dafür, dass Norm und Sachverhalt - oder: Rechtsfrage und Tatfrage - hermeneutisch ${ }^{36}$ untrennbar miteinander verbunden sind. ${ }^{37}$ Richter analysieren Sach-

29 H. Pieper u.a., Sachverständige (Fn. 5), S. 37. Früh und grundlegend zur Rollenverteilung zwischen Richter und Gutachter BGHSt 8, 113. Die Rolle eines medizinischen Sachverständigen ist allerdings deutlich komplizierter. Er ist auch Arzt und an ärztlich-ethische Regeln gebunden. Dazu K. Foerster, Der Verteidiger, sein Mandant und der psychiatrische Sachverständige - eine Dreiecksbeziehung? StV 2008, S. 217 und H. Brettel/H. Vogt, Begutachtung (Fn. 30), S. 20.

30 Bis Mitte des 19.Jahrunderts war die Rechtslage eine andere. Nach römischem Recht und gemeinem Prozessrecht waren Richter an die Gutachten des Sachverständigen gebunden und durften nicht davon abweichen. Ausführlich dazu D. Olzen, Das Verhältnis von Richtern und Sachverständigen im Zivilprozeß unter besonderer Berücksichtigung des Grundsatzes der freien Beweiswürdigung, ZZP 93, 1980, S. 66 (74 f. m.w.N.).

31 So in aller Deutlichkeit - allerdings für den Spezialfall der Prognoseentscheidung - BVerfGE 70, 287 (310).

32 So M. Rehbinder, Rechtssoziologie (Fn. 2), Rn. 162.

33 Die nachfolgenden Ausführungen gelten cum grano salis für alle Sachverständigen, unabhängig von ihrem Fachgebiet.

34 G. Heinz, Fehlerquellen forensisch-psychiatrischer Gutachten, Heidelberg 1982 S. $101 \mathrm{f}$.

35 Dazu am Beispiel des $\mathbb{2} 20$ StGB BGH StraFo 2006, 339.

36 Logisch und sprachphilosophisch lässt sich beides dagegen ohne weiteres trennen. So zu Recht $B$. Schünemann, Kognition, Einstellung und Vorurteil bei der Rechtsfindung, ARSP-Beiheft 22/1985, S. 68 .

37 J. Hruschka, Das Verstehen von Rechtstexten, München 1972, S. 70 f. 
verhalte nicht isoliert für sich, sondern immer im Hinblick auf konkrete Normen; gleichzeitig legen sie Normen immer vor dem Hintergrund eines konkreten Sachverhalts aus. Und Gutachter beschränken sich nicht auf die Analyse der Tatsachen. Anders als es den Anschein hat, werten und urteilen sie auch. ${ }^{38}$ In Wirklichkeit lassen sich kognitiv-rationale Informationsbeschaffung und Analyse einerseits und normativ-wertende Entscheidung nicht trennscharf unterscheiden.

\section{Normative Tätigkeit von Gutachtern}

Der erste Anschein trügt. Sachverständige explorieren nicht nur die Tatsachen; sie werten und urteilen auch. ${ }^{39}$ Sie ziehen nicht nur naturwissenschaftlich begründete Schlüsse aus den von ihnen erforschten Tatsachen. Sie bewerten diese Tatsachen auch und geben damit ein normatives Votum ab. ${ }^{40}$

Das wird besonders deutlich im Strafprozess. Die Frage der Schuldfähigkeit in $\$ 20$ StPO wird auf zwei Ebenen beantwortet. Schuldunfähigkeit kann gegeben sein, wenn eines der vier Merkmale der Schuldausschließung vorliegt. Das ist eine medizinischpsychiatrische Frage, die vom Sachverständigen auf einer mehr oder weniger naturwissenschaftlich fundierten Basis beantwortet wird. ${ }^{41}$ Hinzu kommt aber eine zweite Dimension, die ebenfalls geklärt werden muss: Hatte der Täter die Fähigkeit, das Unrecht seiner Tat einzusehen und nach dieser Einsicht zu handeln? ${ }^{42}$ Das ist keine naturwissenschaftliche Frage mehr, sondern eine normative Wertung. Ein Sachverständiger, der die Schuldfähigkeit eines Angeklagten begutachtet, macht also zweierlei: Er stellt eine medizinische Diagnose, und er gibt eine normative Wertung ab. ${ }^{43}$ Beides lässt sich nicht trennscharf unterscheiden und geht ineinander über. ${ }^{44}$

Ganz ähnlich ist die Situation im Sozialrecht. Weite Bereiche des Sozialrechts beruhen auf Tatbeständen, deren Voraussetzungen nur mit Hilfe medizinischen Sachverstandes geklärt werden können. ${ }^{45}$ Es ist deshalb wenig erstaunlich, dass in etwa $50 \%$ aller Sozialgerichtsverfahren medizinische Gutachter beteiligt sind. ${ }^{46}$ Medizinische Gutachter müssen bei ihrer Arbeit vor den Sozialgerichten mit medizinisch angerei-

38 A. Kaufmann, Das Problem der Abhängigkeit des Strafrichters vom medizinischen Sachverständigen, JZ 1985, S. 1066.

39 A. Kaufmann, Problem (Fn. 39), S. 1066.

40 M. Rehbinder, Rechtssoziologie, Rechtssoziologie (Fn. 2). Rn. 162 m.w.N. A. Kaufmann, Problem (Fn. 39), S. 1066.; G. Heinz, Fehlerquellen (Fn. 35), S. 99; R. Hepp, Sach- und Bewertungsfragen an den medizinischen Sachverständigen im Rechtsstreit, NZV 2013, 581 (582 f.) zeigt das am Beispiel einer einfachen HWS-Distorsion (Schleudertrauma).

41 Dazu im Detail aus psychiatrischer Sicht N. Konrad/W. Rasch, Psychiatrie (Fn. 42), S. 225 ff. m.w.N.

42 Zum „psychisch-normativen“ Aufbau der $\mathbb{\$} \$ 20,21$ StPO G. Tondorf/B. Tondorf, Psychologische und Psychiatrische Sachverständige im Strafverfahren, 3. Aufl. Heidelberg 2011, Rn. 21 m.w.N.

43 Details bei N. Konrad/W. Rasch, Psychiatrie (Fn. 42), S. 230 f. m.w.N.

44 Ähnlich M. Rehbinder, Rechtssoziologie (Fn. 2), Rn. 162 und auch K. Foerster, Der psychiatrische Sachverständige zwischen Norm und Empirie, NJW 1983, S. 2049 (2052 f. m.w.N.). Anders wohl A. Nack, Abhängigkeit des Richters vom Sachverständigen, GA 2009, S. 201 (204 f.).

45 A. Ehlers u.a. (Hrsg.), Medizinisches Gutachten im Prozeß, 3. Aufl. München 2005, Rn. 194. 
cherten Rechtsbegriffen wie „Erwerbsfähigkeit“, ${ }^{47}$ „gesundheitliche Schädigung“ oder „Behinderung“ umgehen. ${ }^{48}$ Das sind keine Begriffe, die sich quasi mathematisch-naturwissenschaftlich mit Inhalt füllen ließen. ${ }^{49}$ Ohne Wertungen lässt sich mit ihnen nicht arbeiten. Es reicht nicht, eine Diagnose zu stellen. Der Gutachter muss auch die Krankheitsfolgen im sozialrechtlichen Kontext herausarbeiten. ${ }^{50}$ Das gilt auch für die rentenrechtlich relevanten Fragen nach der Minderung der Erwerbsfähigkeit im Bundesversorgungsgesetz ${ }^{51}$ und des Grades der Behinderung im Schwerbehindertengesetz. ${ }^{52}$ Hier hat der ärztliche Gutachter einen großen Beurteilungsspielraum. ${ }^{53}$

Gilt das auch für naturwissenschaftlich-technische und ingenieurwissenschaftliche Sachverständige? Auf den ersten Blick ist hier der wertende Anteil bei der Gutachtertätigkeit deutlich geringer. ${ }^{54}$ Bei Baugutachten, handwerklichen, technischen, toxikologischen, chemischen o.ä. Gutachten beschränkt sich die Gutachtertätigkeit scheinbar - weit gehend auf die kognitive Analyse. Beurteilungsspielräume und Wertungsmöglichkeiten scheint es nicht zu geben. Schaut man genauer hin, entpuppt sich diese Sicht als Irrtum. Das hat zwei Gründe. Auch die exakten Naturwissenschaften kommen nicht ohne Unschärfen und Wahrscheinlichkeiten aus, die letztlich abgewogen und bewertet werden müssen. ${ }^{55}$ Daneben wird auch das Technik-und Umweltrecht zunehmend von unbestimmten Rechtsbegriffen dominiert, die naturwissenschaftlich-technisch und gleichzeitig normativ-wertend mit Inhalten gefüllt werden müssen. ${ }^{56}$

\section{Widersprüchliches Recht - Unklare Rollenverteilung im Gerichtssaal}

Das Verfassungsrecht gibt den Richtern eindeutig das Rechtsprechungsmonopol. Gutachter sind Berater und Gehilfen des Gerichts. Nach dem Willen der Verfassung

46 Rehbinder, Rechtssoziologie (Fn. 2)Rn. 23. G. Wannagat, Der Sachverständige im sozialgerichtlichen Verfahren, SGB 1987, S. 349 (350) geht sogar noch von höheren Zahlen aus.

47 Ausführlich zu diesem Begriff aus medizinischer und rechtlicher Sicht H. Brettel/H. Vogt, Begutachtung (Fn. 30), S. $47 \mathrm{ff}$.

48 A. Ehlers u.a. (Hrsg.), Gutachten (Fn. 46), Rn. 194 m.w.N.

49 H. Brettel/H. Vogt, Begutachtung (Fn. 30), S. 48, warnen denn auch davor, bei der Begutachtung „abstrakte Vorgaben mechanisch zur Anwendung zu bringen“.

50 Zur Krankheitsfolgenanalyse im Detail H. Brettel/H. Vogt, Begutachtung (Fn. 30), S. 65 ff. m.w.N.

51 Dazu im Detail H. Kater, Gutachten (Fn. 24)Gutachten (Fn. 24), S. 118 ff. m.w.N.

52 A. Ehlers u.a. (Hrsg.), Gutachten (Fn. 46) Rn. 194 m.w.N.

53 K. Louven, Die Abhängigkeit des Richters der Sozialgerichtsbarkeit von ärztlichen Sachverständigen, DRiZ 1988, S. 241 (244).

54 So ähnlich A. Kaufmann, Problem (Fn. 39), S. 1066.

55 Ein instruktives Beispiel aus dem ingenieurwissenschaftlichen Bereich schildert W.Brötz, Struktur und Inhalt der Aussagen wissenschaftlich-technischer Sachverständiger bei der Bewertung des sicherheitstechnischen Gesamtkonzepts technischer Anlagen, in: F. Nicklisch.u.a. (Hrsg.), Die Rolle des wissenschaftlich-technischen Sachverstandes bei der Genehmigung chemischer und kerntechnischer Anlagen, Heidelberg 1982, S. 104 f.

56 Ausführlich dazu s.u. C.III.1. 
verbleibt die normative Entscheidung aber beim Gericht. Das Rechtssystem ist bei dieser Frage aber widersprüchlich. Denn es gibt Entscheidungen oberster Gerichte, durch die das normative Monopol der Richter aufgebrochen wird. Hier liegen rechtsimmanente Ursachen für den (über)großen Einfluss der Gerichtsgutachter.

\section{Gesetzestechnik: Fachregel als Rechtsregel}

Das moderne Recht arbeitet oft mit unbestimmten Rechtsbegriffen, die durch technisch-naturwissenschaftliche Regeln gefüllt werden sollen. Der Gesetzgeber versucht durch diese Begriffe, das Gesetz mit dem aktuellen Wissensstand des Fachgebiets dynamisch zu verknüpfen. Dadurch soll das Recht dem rasanten Innovationstempo in der Technik und den Naturwissenschaften inhaltlich gewachsen sein. Paradebeispiele dafür sind etwa die „allgemein anerkannten Regeln der Technik “, 57 der „Stand der Technik“, 58 die „,beste verfügbare Technik“ in der Industrieemissionsrichtlinie der EU, ${ }^{59}$ der „Stand von Wissenschaft und Technik“ “60 und die „Regeln der Kunst“ in der Medizin. ${ }^{61}$ Die Folge für die Rechtsanwendung: Der Tatbestand dieser Normen kann nicht mehr ohne technisch-naturwissenschaftliche Kenntnisse ermittelt werden. Reine Rechtskenntnisse reichen nicht mehr aus. Schon um den Tatbestand einer Norm genauer zu erfassen, muss der Richter technisch-naturwissenschaftliche Sachverständige befragen.

Diese Gesetzestechnik erzwingt den exzessiven Einsatz von Sachverständigen vor Gericht. Ob eine rechtliche Regelung dann wirksam wird, ist keine Rechtsfrage mehr, sondern eine technisch-naturwissenschaftliche Sachfrage. Dadurch wird die Fachregel zur Rechtsregel. Der Gutachter entscheidet die Fachfrage - und damit automatisch die Rechtsfrage. Der Richter hat dann idR keinen eigenen Spielraum mehr. ${ }^{62}$ Eine Dominanz des Technischen vor dem Juristischen ist die Folge.

Hier werden zwei eng verbundene Gründe für die starke Stellung der Gutachter im Gerichtsverfahren deutlich. Die Gesetze benutzen Schlüsselbegriffe, die letztlich kein Richter ohne Hilfe von Sachverständigen klären kann. Gleichzeitig sind diese Begriffe so strukturiert, dass kein Sachverständiger sie rein kognitiv und naturwissenschaftlich mit Leben füllen kann. Die vom Gesetzgeber verwandten Termini erfordern zusätzlich eine Wertung des Gutachters. Vor diesem Hintergrund erklärt sich, dass

57 Z.B. $\$ 2$ EBO oder die Regelungen in den Landesbauordnungen der Länder.

58 Z.B. $\$ 3$ Abs. 6 BImSchG, $\$ 57$ Abs. 2 WHG;.

59 Art. 3 Nr. 10, Richtlinie 2010/75/EU.

60 Z.B. $\mathbb{\int} 12 \mathrm{a}, 18 \mathrm{TFG}$; $\mathbb{7}$ Abs. 2 Nr. 3 AtG.

61 Z.B. $\int 630$ a BGB.

62 Ähnlich schon W. Sass, Der Sachverständige - weiterhin ein prozessuales Problemfeld, DS 2007, S. 256 (257). 
Gerichte in der weit überwiegenden Zahl der Fälle dem Gutachter folgen ${ }^{63}$ - nicht selten sogar ohne eigene Reflexionen. ${ }^{64}$

Lässt sich das ändern, um die Stellung des Richters im Prozess wieder zu stärken? Diese Entwicklung des Rechts wird sich nicht rückgängig machen lassen. Der Preis dafür wäre wohl zu hoch. Ohne offene Rechtsbegriffe, die technisches und naturwissenschaftliches Know-how in das Rechtssystem inkorporieren, lässt sich kein Recht schaffen, das moderne Technik dynamisch und angemessen steuern könnte. ${ }^{65}$

\section{Rechtsprechung: Bindung an das Gutachten als Regel}

Die präjudizierende Macht der Gutachter beruht auch auf einer Rechtsprechung des Bundesverfassungsgerichts und des Bundesgerichtshofs. Das BVerfG betont in inzwischen ständiger Rechtsprechung, dass Gerichte nur unter bestimmten, strengen Voraussetzungen von den fachlichen Feststellungen und Wertungen eines Sachverständigengutachtens abweichen dürfen. ${ }^{66}$ Ein Abweichen ist nur zulässig, wenn das Gericht eigene Sachkunde hat und nachweist. ${ }^{67}$ In jedem Fall muss das Gericht sein Abweichen eingehend begründen. Sehr ähnlich ist schon lange die Judikatur des Bundesgerichtshofs. Gerichte, die von Gutachten abweichen, müssen die Einzelheiten des Gutachtens und ihre Gründe für das Abweichen ausführlich und fundiert erörtern. ${ }^{68}$ Noch weiter geht die Rechtsprechung zum Beweiswert von standardisierten Sachverständigengutachten wie etwa Blutgruppengutachten, ${ }^{69}$ Blutalkoholanalysen ${ }^{70}$ oder daktyloskopische Gutachten. In ständiger Rechtsprechung hat sich der Bundesgerichtshof auf den Standpunkt gestellt, dass im Hinblick auf deren Beweiswert jegliche richterliche Würdigung unterbleiben kann. ${ }^{71}$ Es reicht, wenn der Richter das Ergebnis referiert und sich diesem Ergebnis anschließt. ${ }^{72}$ Diese Rechtsprechung passt in den bereits skizzierten Trend zur Verwissenschaftlichung. Die Freiheit der richterlichen Beweiswürdigung bedeutet danach nicht, dass ein Richter

63 B. Jordan/U. Gresser, Gutachter (Fn. 9), S. 71 (75) mit empirischem Material.

64 So zum Strafprozess K. Foerster, FS Schreiber (Fn. 4), S. 82 f.; zum sozialgerichtlichen Verfahren K. Foerster, in: FS H.-L. Schreiber, Heidelberg 2003, S. 83 f.; zum Arzthaftungsprozess C. Katzenmeier, in: A. Laufs/C. Katzenmeier/V. Lipp, Arztrecht, 6. Aufl. München 2009. Kap. XII. A. Rn. 7 m.w.N.; zum Zivilprozess H. Pieper u.a., Sachverständige (Fn. 5), S. 261 ff. auf der Grundlage einer empirischen Untersuchung.

$65 \mathrm{Ob}$ Recht überhaupt Technik steuern kann, ist heftig umstritten. Ausführlich dazu Boehme-Neßler, Unscharfes Recht, Berlin 2008, S. 62 ff. m.w.N.

66 BVerfG NJW 1999, 3623, 3624; BVerfG, 1 BvR 971/03 vom 24.7.2006, Absatz-Nr. 15, http:// www.bverfg.de/entscheidungen/rk20060724_1bvr097103.html (29.7.2013); BVerfG, 1 BvR 1253/06 vom 9.5.2007, Absatz-Nr. 15, http://www.bverfg.de/entscheidungen/rk20070509_1bvr125306.html 29.7.2013).

67 BGH NStZ 2005, 628; BGH NStZ 2000, 437.

68 BGH GA 1977, 275; BGH StV 94, 359; BGH NStZ-RR 2009, 181; BGH NStZ 2010, 106.

69 Grundlegend BGHSt 39, 291 (297ff.).

70 BGHSt 28, 235 (237 f.).

71 P. Arens, 1. Deutscher Landesbericht, in: F. Nicklisch (Hrsg.), Der Technische Sachverständige im Prozess, Heidelberg 1984, S. 32 m.w.N.

72 BGH Beschluss vom 15.9.2010, 5 StR 345/10, Rn. 9. 
wissenschaftliche Erkenntnisse außer Acht lassen darf. ${ }^{73}$ Auf Alltagstheorien darf er nur zurückgreifen, wenn wissenschaftliche Einsichten nicht dagegen sprechen.

Zwar betont das Verfassungsgericht also immer wieder ausdrücklich: Gerichte dürfen grundsätzlich von Sachverständigengutachten abweichen. Es stellt dafür aber hohe Hürden auf. So plausibel die Rechtsprechung der obersten Gerichte scheint, so problematisch ist ihre Auswirkung in der Praxis. Der (revisionsrechtliche) Anreiz für Gerichte, dem Gutachten kritiklos zu folgen, ist unwiderstehlich. ${ }^{74}$ Denn der Aufwand für eine eigene Überzeugung, die dem Gutachter widerspricht, ist (zu) groß. Faktisch ergibt sich daraus eine Bindung des Gerichts an die Meinung des Sachverständigen. ${ }^{75}$

\section{Fazit: Unscharfe Rollenverteilung zwischen Gericht und Gutachter}

Der Verfassung und den Prozessordnungen schwebt eine klare Rollenverteilung zwischen Gericht und Gutachter vor. Schon die Erkenntnistheorie zeigt aber, dass sich beide Rollen nicht trennscharf unterscheiden lassen. Es gibt breite Schnittmengen zwischen ihnen. Schon theoretisch ist es nicht denkbar, dass ein Gerichtsgutachter nur analytisch arbeitet. Dass seine Tätigkeit in der Regel auch normative Elemente enthält, ist eine erkenntnistheoretische Binsenweisheit.

Gerade die Gesetzgebung in den Rechtsgebieten, die besonders auf Sachverständige angewiesen sind, lässt die Rollenverteilung zunehmend unscharfer werden. Im Umweltrecht etwa, im Medizinrecht und im Sozialrecht arbeitete der Gesetzgeber mit offenen, fachwissenschaftlich geprägten Rechtsbegriffen. Das führt dazu, dass fachkundige Sachverständige unentbehrlich. Mit ihren Fachkenntnissen füllen Sie Rechtsbegriffe aus - und arbeiten damit auch normativ.

Die Rechtsprechung hat das pragmatisch akzeptiert. Sie geht - mehr oder weniger ausdrücklich - davon aus, dass Gerichte in der Regel faktisch an die Sachverständigengutachten gebunden sind. Das verleiht den Gerichtsgutachter - höchstrichterlich akzeptiert - faktisch normative Kompetenzen.

Wie unscharf die Grenze und wie unklar die Rollenverteilung zwischen Gerichtsgutachter und Gericht tatsächlich ist, macht ein Blick auf sozialpsychologische Aspekte deutlich.

73 Ähnlich G. Fezer, Tatrichterlicher Erkenntnisprozeß - „Freiheit der Beweiswürdigung“ StV 1995, S. 95 (97ff.).

74 Ähnlich H. Sendler, Richter und Sachverständige, NJW 1986, S. 2907 (2909 m.w.N.), D. Olzen, Das Verhältnis von Richtern und Sachverständigen im Zivilprozeß unter besonderer Berücksichtigung des Grundsatzes der freien Beweiswürdigung, ZZP 93 (1980), S. 66 (79).

75 P. Arens, Landesbericht (Fn. 72), S. 32. 


\section{Richter und Sachverständige - Sozialpsychologische Aspekte einer komplexen Beziehung}

Richter entscheiden nicht im luftleeren Raum. Die Prozessordnungen gehen davon aus, dass Urteile des Gerichts ausschließlich auf dem Diskurs im Gerichtssaal basieren. Selbstverständlich hat eine Gerichtsverhandlung einen großen Einfluss auf die Entscheidung des Gerichts. Dass dieser Einfluss aber exklusiv wäre, ist eine Fiktion. Wie bei allen anderen Menschen auch, werden richterliche Entscheidungen von einer Vielzahl äußerer und innerer Faktoren beeinflusst. Ein - möglichst großer - Anteil der Faktoren ist juristischer Natur. Weithin unterschätzt ist aber, dass auch sozialpsychologische Einflüsse eine große Rolle spielen. ${ }^{76}$

\section{Die Macht der Situation}

Richter und Gutachter treffen in einer speziellen Situation aufeinander: Sie erfüllen unterschiedliche Funktionen in einem gerichtlichen Verfahren. Das beeinflusst ihr Verhalten. Denn Erkenntnisse der Sozialpsychologie belegen, dass menschliches Handeln auch durch die Situation beeinflusst wird, in der es stattfindet.

\section{Sozialpsychologie: Situative Einflüsse auf menschliches Verhalten}

Die Psychologie hat viele empirische Belege dafür gefunden, dass menschliches Handeln stark durch situative Einflüsse (mit)bestimmt wird. In bestimmten sozialen Situationen handeln Menschen anders, als es ihre Überzeugungen, Werte und Dispositionen erwarten lassen. ${ }^{77}$ Die Macht der Situation ${ }^{78}$ wird oft gar nicht wahrgenommen. Auch Richter können sich diesen psychologischen Mechanismen nicht oder nur begrenzt - entziehen. Die Sozialpsychologie hat eine Fülle von Beispielen dafür gefunden, wie Besonderheiten der Situation Einfluss auf richterliche Entscheidungen entwickelt haben. Amerikanische Studien zeigen, dass die Hautfarbe eines Angeklagten oder seine (mangelnde) Attraktivität in vielen Fällen Auswirkungen auf die Höhe der Strafe hat. ${ }^{79}$ Ein anderes Beispiel: Je höher die Strafe, die der Staatsanwalt fordert, desto höher ist - in identischen Fällen - das Strafmaß, das im Urteil festgelegt wird. ${ }^{80}$ Oder: Höhere Schadensersatzforderungen ziehen in identischen

76 B. Englich, Urteilseinflüsse vor Gericht, in: R. Volbert/M. Steller (Hrsg.): Handbuch der Rechtspsychologie, Göttingen 2008, S. 486 (489).

77 Ausführlich dazu P. Zimbardo, Der Luzifer-Effekt, Heidelberg 2008, S. 207 ff., 250 ff. und pass.

78 Grundsätzlich dazu P. Zimbardo/R. Gerrig, Psychologie, 16. Aufl. München 2004, S. 753 ff. m.w.N.

79 B. Englich, „Geben Sie ihm doch einfach fünf Jahre! “ Zeitschrift für Sozialpsychologie 2005, S. 215 (216 m.w.N.) Die Strafen werden auch signifikant höher, wenn die Angeklagten typische afroamerikanische Gesichtszüge aufweisen. Ausführlich dazu I. Blair u.a., The Influence of Afrocentric Facial Features in Criminal Sentencing, Psychological Science 15, 2004, $674 \mathrm{ff}$.

80 B. Englich, Jahre (Fn. 80), S. 215 (217 m.w.N.); B. Englich u.a., The Last Word in Court - A Hidden Disadvantage for the Defense, Law and Human Behavior 2005, S. 705, (706f.); E. Garrido Martin u.a., Influence of the Prosecutor's Plea on the Judge's Sentencing in Sexual Crimes, in: S. Redondo u.a. (Hrsg.), Advances in Psychology and Law, Berlin 1997, S. 215. (221). Kritisch dazu aber aus methodischer Sicht M. Schweizer, Kognitive Täuschungen vor Gericht.(Dissertation) Zürich 2005, S. 81. 
Fallgestaltungen höhere Schadensersatzurteile nach sich. ${ }^{81}$ Selbst Zwischenrufe aus dem Zuschauerraum haben nicht selten Auswirkungen auf das Urteil des Richters. $^{82}$

Ein weiteres, ganz profanes und noch nicht empirisch untersuchtes Beispiel dürfte der Druck des Pensenschlüssels sein, der im richterlichen Berufsalltag eine große Rolle spielt. Wer in immer kürzerer Zeit immer mehr Verfahren „erledigen“ muss, läuft - womöglich ohne sich dessen ganz bewusst zu sein - Gefahr, prozessökonomischen Überlegungen einen zu hohen, rechtsstaatlich bedenklichen Stellenwert einzuräumen.

\section{Informationaler sozialer Einfluss}

Menschen sind grundsätzlich bereit, auf andere zu hören und deren Meinungen über die soziale Wirklichkeit zu übernehmen. ${ }^{83}$ Menschen sind kommunikative, soziale Wesen, die auf den Rückhalt in der Gruppe angewiesen sind. Was andere meinen, ist aus diesem Blickwinkel (überlebens)wichtig (gewesen). Dieser informationale soziale Einfluss wird durch zwei Faktoren noch weiter gesteigert. Je unsicherer man ist, und je wichtiger die richtige Antwort ist, desto größer ist die Bereitschaft, auf andere zu hören. ${ }^{84}$ Hier liegt eine wichtige Wurzel für die große Bereitschaft der Richter, den Gutachtern zu folgen. Sie sind wegen einer fachlichen Frage, die Sie nicht beurteilen können, verunsichert. Gleichzeitig hängt von dieser Frage ab, wie ihr Urteil aussehen wird. Die Antwort auf die Fachfrage ist also extrem wichtig. Der informationale soziale Einfluss des Gutachters auf den Richtern ist deshalb besonders groß.

Hier liegt auch eine sozialpsychologische Ursache für das entgegengesetzte Phänomen: Auch der Gutachter steht in der spezifischen sozialen Situation vor Gericht unter Anpassungsdruck. Nicht selten führt das zu einer Überidentifikation mit der Justiz. Dann besteht die Gefahr, dass sein Gutachten darauf zielt, die Erwartungen des Gerichts zu erfüllen. ${ }^{85}$

\section{Autorität der Experten und des Gerichts}

Gutachter treten mit der Autorität des Experten auf. Ein wichtiges Mittel dazu ist ihre Fachsprache. ${ }^{86}$ Das erhöht ihren faktischen Einfluss im Prozess noch weiter. Denn Menschen gehorchen einer Autorität grundsätzlich. ${ }^{87}$ Die Gründe dafür sind

81 B. Englich u.a., Playing Dice With Criminal Sentences, Personality and Social Psychology Bulletin 2006, S. 188 (189 m.w.N.); G. Chapman u.a., The more you ask for, the more you get, Applied Cognitive Psychology 10, 1996, S. 519 (520 ff.).

82 B. Englich, Jahre (Fn. 80), S. 215 (218, 221 ff.) mit umfangreichem empirischem Material.

83 D. Myers, Psychologie, 2. Aufl. Berlin 2008, S. 647 m.w.N.

84 D. Myers, Psychologie (Fn. 84), S. 647 f. m.w.N.

85 Ausführlich dazu s. u. E.

86 A. Kaufmann, Problem (Fn. 39), S. 1070.

87 P. Zimbardo, Luzifer (Fn. 78), S. 264 ff.; bahnbrechend dazu S. Milgram, Obedience to Authority, London: Tavistock 1974. 
vielfältig. ${ }^{88}$ Eine wichtige Rolle spielt die Macht sozialer Billigung. ${ }^{89}$ Menschen haben das starke Bedürfnis, sozial akzeptiert und geschätzt zu werden. Dafür tun sie (fast) alles, was Autoritäten von ihnen wollen. Genauso relevant ist letztlich die Sozialisation der meisten Menschen: Von Kindesbeinen an lernen sie, einer Autorität zu gehorchen, ohne Fragen zu stellen. ${ }^{90}$ Von diesen Einflüssen können sich auch Richter nicht (vollständig) freimachen. Auch sie werden mit der Autorität des Experten in ihrem Gerichtssaal konfrontiert und müssen sich damit auseinandersetzen. Hinzu kommt, dass Richter Ängste überwinden müssen, wenn sie mit Gutachtern kritisch über Gutachten diskutieren: Ängste, sich als Laie erkennen zu geben, ihre Autorität als Richter zu verlieren oder sich sogar zu blamieren. Dann ist die Versuchung groß, diese Gefahr zu vermeiden - und das Gutachten kritiklos hinzunehmen.

Ähnlich schwierig ist auch die Situation des Sachverständigen. Wenn er den Gerichtssaal betritt, wird er mit der Autorität der Justizpersonen konfrontiert. Er ist nicht mehr der unangefochtene - vielleicht sogar bewunderte - Experte in seiner gewohnten Umgebung. Er bewegt sich auf fremdem Terrain. Er wird kritisch, bisweilen scharf und unhöflich von Staatsanwälten, Rechtsanwälten und Richtern befragt. Seine Autorität als Experte wird ausdrücklich oder unterschwellig nicht selten in Frage gestellt. Das ist eine Situation, die sehr einschüchtern kann. Sie birgt die Gefahr, dass die Autorität des Gerichtssaals den Gutachter einschüchternd. Die fatale Folge kann sein: er wechselt die Rolle. Vom objektiven unabhängigen Experten und Berater des Gerichts wird er zum Gehilfen, der Erwartungen erfüllen will.

\section{Fazit: Bewusstsein und Respekt}

Die Situation, in der sich Sachverständige und Richter vor Gericht begegnen, ist schwierig. Beide Seiten laufen Gefahr, sich von der Autorität der anderen Seite einschüchtern zu lassen. Das darf allerdings nicht passieren. Denn dann können beide - Gutachter und Richter - ihre anspruchsvollen Rollen im Prozess nicht erfüllen. Entscheidend ist: Beide müssen sich der Problematik der Situation bewusst sein und den jeweils anderen in seiner unterschiedlichen Rolle respektieren. Dann funktioniert der notwendige, aber schwierige Kommunikationsprozess zwischen Gericht und Gutachter.

\section{Verzerrende Urteilsheuristiken}

Menschen treffen Entscheidungen eher selten nach langen, fundierten und komplexen Überlegungen. Sie entscheiden im Zweifel schnell mit Hilfe von Heuristiken. Heuristiken sind ein probates Mittel, um schnelle Entscheidungen zu treffen, ohne alle relevanten Informationen zu kennen. Genau das ist die klassische Entschei- 
dungssituation im Alltag - und im Gerichtssaal. Deshalb sind Heuristiken auch ein wichtiges Mittel von Richtern, um Entscheidungen zu treffen und Urteile zu fällen.

So hilfreich und wichtig Heuristiken sind: Sie sind auch gefährlich. Sie verzerren den Blick auf die Wirklichkeit und können zu falschen Entscheidungen führen.

\section{Unverzichtbar - (Urteils)Heuristiken als Instrumente des Denkens}

So komplex das menschliche Gehirn auch ist: im Vergleich zur Komplexität der Umwelt ist das menschliche Denkvermögen sehr bescheiden. ${ }^{91}$ Menschen müssen sich damit zufrieden geben, Problemlösungen zu finden, die „gut genug“ sind. ${ }^{92}$ Die optimale Lösung - wenn sie überhaupt existiert - lässt sich in der Regel kaum finden. Heuristiken sind unverzichtbare Instrumente für diese Art des menschlichen Denkens. ${ }^{93}$ Mit Hilfe solcher mentalen Faustregeln lassen sich schnell Lösungen finden, die selten optimal, aber in der Regel sehr praktikabel sind.

In der Kognitionspsychologie werden drei große Gruppen von Heuristiken unterschieden, mit deren Hilfe schnell akzeptable Urteile in komplexen Situationen gefällt werden können. ${ }^{94}$ Die Verfügbarkeitsheuristik bedeutet: Menschen gründen ihr Urteil meistens auf Informationen, die im Gedächtnis leicht und schnell verfügbar sind. ${ }^{95}$ Das macht schnelle Urteile möglich, ohne dass komplexe Informationen verarbeitet und abgewogen werden müssten. Ähnlich ist der Vorteil der Repräsentativitätsheuristik. ${ }^{96}$ Sie bewertet die Wahrscheinlichkeit von Ereignissen danach, wie sehr sie zu einem bestimmten Prototypen passen. ${ }^{97}$ Von großer Bedeutung ist schließlich die Ankerheuristik. ${ }^{98}$ Eine bestimmte Information am Anfang eines Entscheidungsprozesses prägt und beeinflusst überproportional das nachfolgende Urteil. Es orientiert sich an ihr wie an einem Anker.

Diese Heuristiken haben sich im Lauf der menschlichen Evolution entwickelt. Sie sind ein unverzichtbares Instrument, um Entscheidungen in hochkomplexen, unübersichtlichen Situationen auf mangelhafter Datenbasis und unter Zeitdruck zu

91 H.A. Simon, Models of Thought, New Haven, CT: Yale University Press, 1979, S. 3.

92 H.A. Simon, Models (Fn. 92), S. 3.

93 P. Zimbardo/R. Gerrig, Psychologie (Fn. 89), S. $311 \mathrm{f}$.

94 Grundlegend dazu A. Tversky/D. Kahneman, Judgment under Uncertainty: Heuristics and Biases, in: Science Vol. 185, 1974, S. 1124.

95 P. Zimbardo/R. Gerrig, Psychologie (Fn. 89), S. 313. Bahnbrechend dazu A. Tversky/D. Kahneman, Availability: A heuristic for judging frequency and probability, in: Cognitive Psychology, Vol. 5 1973, S. $207 \mathrm{ff}$.

96 P. Zimbardo/R. Gerrig, Psychologie (Fn. 89), S. 314 f. Grundlegend im Detail A. Tversky/D. Kahneman, Judgment (Fn. 95), S. 1124.

97 Ein Beispiel dafür: Rechtsanwälte spielen meistens Tennis oder Golf. Also wird die Lieblingssportart von Herrn Rechtsanwalt A Tennis oder Golf sein. In vielen - aber nicht in allen - Fällen liegt man mit dieser Heuristik richtig.

98 P. Zimbardo/R. Gerrig, Psychologie (Fn. 89), S. 315 f. 
treffen. Heuristiken führen aber - und das ist eine große Gefahr - leicht zu kognitiven Verzerrungen. ${ }^{99}$

Heuristiken sind für die praktische Arbeit der Justiz von großer Bedeutung. Das heißt allerdings auch: Die kognitiven Verzerrungen, mit denen die Heuristiken oft verbunden sind, werden zum Problem für das Prozessrecht und den Rechtsstaat.

\section{Verfügbarkeits- und Repräsentativitätsheuristik im Gerichtssaal}

In der Justiz wird unter hohem Zeitdruck entschieden. ${ }^{100}$ Ohne Heuristiken ist das nicht zu schaffen. Vor allem die Verfügbarkeits-und Repräsentativitätsheuristik helfen den Richtern, ihr Entscheidungspensum zu bewältigen.

\section{a) Verfügbarkeitsheuristik}

Ein großer Teil der richterlichen Tätigkeit besteht darin, Einschätzungen auf oft unzureichender Informationsgrundlage vorzunehmen. Richter müssen permanent einschätzen: Personen, Situationen, Aussagen, Verhaltensweisen und Argumente. Wie alle Menschen tun sie das mit Hilfe der Verfügbarkeitsheuristik. ${ }^{101}$ Wie Sie jemanden oder etwas einschätzen, hängt von dem Wissen ab, das ihnen leicht und schnell etwa aus ihrem Erfahrungsschatz oder ihrem Gedächtnis - verfügbar ist. Das ist kein komplexes, wissenschaftlich fundiertes Wissen. Das sind die sog. Alltagstheorien, mit denen letztlich jeder Mensch arbeitet. ${ }^{102}$ Ein Beispiel dafür sind die richterlichen Mutmaßungen über die Verkehrsauffassung. ${ }^{103}$ Wie die Verkehrsauffassung ist, klären Gerichte meist selbst, ohne nähere Untersuchungen und tiefere Überlegungen. Die Gefahr ist groß, dass ein Gericht seine eigene, subjektive Auffassung als allgemeine Verkehrsauffassung deklariert. ${ }^{104}$

Gerichte haben nicht die Zeit, alle relevanten Fakten wissenschaftlich einwandfrei zu beschaffen und zu bewerten. Ohne die Verfügbarkeitsheuristik und ihre Alltagstheorien wären Richter kaum in der Lage, ihr Arbeitspensum zu bewältigen. Das Problem dabei: Die Wirklichkeit wird nicht, nur teilweise oder verzerrt zur Kenntnis genommen. Stattdessen wird sie durch plausible, aber oft falsche oder unzulängliche

99 Im Detail dazu P. Zimbardo/R. Gerrig, Psychologie (Fn. 89), S. 312 ff. m.w.N. und A. Tversky/D. Kahneman, Judgment (Fn. 95), S. 1124.

100 Dazu M. Dölp, Der Sachverständige im Strafprozess, ZRP 2004, S. 235 Fn. 1; R. Lamprecht, Außerrechtliche Einflüsse auf die richterliche Entscheidungsfindung DRiZ 1989, S. 4 (7); R. Lautmann, Justiz - Die stille Gewalt. Frankfurt a. M. 1972, S. 49.

101 Zur Verfügbarkeitsheuristik ausführlich D. Kahneman, Schnelles Denken, langsames Denken, Berlin 2012, S. $164 \mathrm{ff}$.

102 Eine Fülle von unterschiedlichen Alltagstheorien, mit denen Richter arbeiten, hat J. Bürkle, Richterliche Alltagstheorien im Bereich des Zivilrechts. Tübingen 1984, S. 17 ff. und pass. zusammengetragen. Schon früher R. Lautmann, Gewalt (Fn. 101), S. $57 \mathrm{ff}$.

103 T. Müller, Mutmaßungen über die Verkehrsauffassung, JR 1992, S. 8 (9).

104 T. Müller, Mutmaßungen (Fn. 105), S. 8 (9). 
Annahmen über die Wirklichkeit ersetzt. ${ }^{105}$ Hier liegt eine potentielle Ursache für schlechte oder falsche Urteile.

\section{b) Repräsentativitätsheuristik}

Ein vergleichbares Problem ist mit der Repräsentativitätsheuristik ${ }^{106}$ verbunden. Sie ist eine weitere Technik, mit deren Hilfe Menschen versuchen, Informationen zu kategorisieren und Wahrscheinlichkeiten richtig einzuschätzen. Wahrscheinlichkeitsurteile sind sehr schwierig. Menschliches Denken nutzt deshalb die Repräsentativität als Hilfsmittel. ${ }^{107}$ Anhand von Merkmalen, die scheinbar repräsentativ sind, wird ein Mensch, eine Situation oder eine Entwicklung eingeschätzt. Weil etwas in einer Hinsicht ähnlich ist, wird es deshalb wahrscheinlich auch in anderer Hinsicht genauso sein. Ein plastisches Beispiel dafür: Weil ältere Rechtsanwälte oft Golf spielen, wird Herr A, der auch Rechtsanwalt in den besten Jahren ist, wohl auch gerne Golf spielen.

Dieses intuitive und stereotype Denken ist im Alltag effektiv. Sein Ergebnis sind Einschätzungen, die in aller Regel besser als reine Zufallsergebnisse sind. ${ }^{108}$ Trotzdem führt auch diese Heuristik oft zu Fehleinschätzungen. Denn sie widerspricht der statistischen Logik. ${ }^{109}$ Und sie öffnet Stereotypen und Vorurteilen Tür und Tor.

Für die Entscheidungsfindung vor Gericht ist das problematisch. Die Repräsentativitätsheuristik ist ein übliches Mittel bei der Ermittlung des Sachverhalts, um fehlende Fakten in kürzester Zeit zu ergänzen. Gleichzeitig ist sie fehleranfällig und verzerrt den Blick auf die Wirklichkeit. Das macht sie - wie die Verfügbarkeitsheuristik - zu einer potentiellen Ursache für schlechte oder falsche Entscheidungen von Gerichten.

\section{c) Alltagstheorien in der Gerichtspraxis}

Problematisch wird es immer dann, wenn die Rechtsprechung die (allgemeine) Lebenserfahrung und die eigene richterliche Sachkunde als Argument heranzieht. Das geschieht relativ oft, wenn Fakten und Zusammenhänge (scheinbar) klar sind und weitere Ermittlungen deshalb (scheinbar) nicht mehr nötig sind. ${ }^{110}$ Ist es dann die wirkliche, empirisch gestützte Lebenserfahrung, von der die Richter ausgehen? Oder ist es die allgemeine Lebenserfahrung, wie sie die Richter mit Hilfe der Verfügbar-

105 Beispiele dafür bei R. Lautmann, Gewalt (Fn. 101), S. 55 ff.; M. Rehbinder, Abhandlungen zur Rechtssoziologie, Berlin 1995, S. 215 nennt das treffend „Gefühlsjurisprudenz“.

106 Ausführlich dazu D. Kahneman, Schnelles Denken, langsames Denken, Berlin 2012, S. 187 ff. m.w.N.

107 D. Kahneman, Denken (Fn. 102), S. 189 spricht bildhaft von einer „mentalen Schrotflinte“.

108 D. Kahneman, Denken (Fn. 102), S. 190.

109 D. Kahneman, Denken (Fn. 102), S. 190 ff. mit instruktiven Beispielen.

110 Ein schönes Beispiel dafür ist $S$. Broß, Richter und Sachverständiger, dargestellt anhand ausgewählter Probleme des Zivilprozesses, ZZP 1989 Vol. 102, S. 413 (417). Er behauptet, dass bei technischen Fragen Richter aufgrund ihrer Lebens-und Berufserfahrung besonders sachverständig seien. 
keits-und Repräsentativitätsheuristik fingiert haben? Auch an dieser Weichenstellung entscheidet sich, ob die Entscheidung eines Gerichts gut oder schlecht ist.

Andere Beispiele finden sich im Zivilrecht. Ob eine Eigenschaft verkehrswesentlich oder ein Fehler nach der Verkehrsanschauung erheblich ist, hängt davon ab, welche Vorstellung die beteiligten Verkehrskreise haben. Die gerichtliche Praxis entscheidet das oft ohne die tatsächlichen Vorstellungen der beteiligten Verkehrskreise wirklich zu ermitteln. ${ }^{111}$ Mit anderen Worten: Die Richter greifen auf die Verfügbarkeits- und der Repräsentativitätsheuristik zurück, um diese Fragen zu beantworten. ${ }^{112}$ Das kann - wie immer bei Heuristiken - im Einzelfall zu einer richtigen Entscheidung führen. Die Gefahr ist aber groß, dass die Richter dabei nicht die wirklichen Vorstellungen der beteiligten Verkehrskreise ermitteln, sondern sie selber unbewusst definieren.

Eine ähnliche Problematik existiert im Markenrecht. Ein Schlüsselbegriff ist die Verwechslungsgefahr einer Marke. Entscheidend dafür sind die Vorstellungen, die das Publikum von der Marke hat. Sehr oft entscheiden Gerichte über die Verwechslungsgefahr auf der Grundlage allgemeiner Lebenserfahrung und eigener Sachkunde. Das kann - mehr oder weniger zufällig - zu einem richtigen Ergebnis führen. Die Gefahr ist aber, dass die Richter ihre eigene - materiellrechtlich irrelevante - Vorstellung von der Verwechslungsgefahr als die Vorstellung der beteiligten Verkehrskreise ausgeben. ${ }^{113}$

\section{Der Ankereffekt im Gerichtssaal}

Ein weiteres Beispiel für eine Heuristik, die ein Urteil verzerren kann, ist der Ankereffekt. ${ }^{114}$ Unter einem Anker versteht die Kognitionspsychologie eine - auch völlig willkürliche -Information, die sich darauf auswirkt, wie eine Situation eingeschätzt und eine Entscheidung getroffen wird. ${ }^{115}$ Völlig unabhängig davon, wie sinnvoll die Information ist: Sie beeinflusst die Entscheidung. Empirisch lässt sich nachweisen, dass der Ankereffekt auch bei Gerichtsurteilen wirkt. ${ }^{116}$

111 Rehbinder, Rechtssoziologie (Fn. 2)Rn. 9 m.w.N.

112 Wie Rehbinder, Rechtssoziologie (Fn. 2), Rn. 9 m.w.N., betont, scheint sich im Recht des gewerblichen Rechtsschutzes eine Abkehr von dieser Praxis anzubahnen.

113 Sehr kritisch dazu M. Rehbinder, Abhandlungen zur Rechtssoziologie. Berlin 1995, S. 210 f. m.w.N.

114 B. Englich, Urteilseinflüsse (Fn. 77), S. 486 m.w.N.

115 Bahnbrechend dazu A. Tversky/D. Kahneman, Judgment (Fn. 95), S. 1124 (1128 ff.) Ausführlich zu den Ursachen dieses Effekts T. Mussweiler/F. Strack, Comparing Is Believing: A Selective Accessibility Model of Judgmental Anchoring, European Review of Social Psychology 1999, Vol. 10, S. 135 (145 ff.) auf der Basis umfangr. empirischen Materials.

116 B. Englich, Urteilseinflüsse (Fn. 77), S. 486 ff. m.w.N. 
Empirisch gut untersucht ist, welche (deutlichen) Auswirkungen die Höhe zivilrechtlicher Klagen ${ }^{117}$ oder die Strafforderungen im Plädoyer von Staatsanwälten ${ }^{118}$ auf die Urteile von Gerichten haben. Beides - die eingeklagte Summe und die vom Staatsanwalt geforderte Strafe - sind wirksame Anker, die Urteile deutlich beeinflussen. Die Fragen von Journalisten etwa oder explizit zufallsgenerierte Strafanträge von Staatsanwälten zeigen Ankereffekte auf die Urteile. ${ }^{119}$ Das gilt sogar für offensichtlich unqualifizierte, parteiische Zwischenrufe im Gerichtssaal. ${ }^{120}$

Empirische Studien zeigen, dass es äußerst schwierig ist, sich dem Ankereffekt zu entziehen. ${ }^{121}$ Er entfaltet sogar dann Wirkung, wenn man sich seiner bewusst ist und ausdrücklich vor ihm gewarnt wird. ${ }^{122}$ dieses sozialpsychologische Forschungsergebnis ist relevant für eine Theorie richterlichen Entscheidens.

In einem Urteil dürfen grundsätzlich nur die Informationen verwendet werden, die im Verfahren gesammelt worden sind. Das ist eine Grundidee der Prozesstheorie. Besonders deutlich wird sie in $\mathbb{} 261$ StPO formuliert, der vom „Inbegriff der Verhandlung “ als Grundlage der gerichtlichen Entscheidung spricht. Angesichts des Ankereffekts ist das grundsätzlich eine sehr sinnvolle Idee. Sie ist der Versuch, urteilsverzerrende Auswirkungen irrelevanter, willkürlicher Informationen zu vermeiden. Allerdings reicht das nicht aus. Es gibt zu viele Möglichkeiten, Ankereffekte zu setzen, die ein Urteil dann verzerren können. Wichtig wäre es, dass sich alle Richter dieses Effekts und seiner verzerrenden Wirkungen bewusst sind. Dann könnten sie ihm entgegenwirken und ihn - mehr oder weniger - ausschalten. ${ }^{123}$

\section{Heuristiken und Gerichtsgutachten}

Heuristiken spielen im Gerichtsalltag also eine sehr ambivalente Rolle. Sie erleichtern - vielleicht sogar: ermöglichen - die Arbeit in der Gerichtspraxis. Gleichzeitig stellen Sie aber eine unterschätzte und sehr problematische Quelle für Fehler in Urteilen und Beschlüssen darf. Hier können Gerichtsgutachten sehr helfen, Heuristiken durch

117 Bahnbrechend G. Chapman u.a., The more you ask for, the more you get, Applied Cognitive Psychology 10, 1996, S. 519; Ausführlich dazu R. Hastie et al., Juror Judgments in Civil Cases: Effects of Plaintiff's Requests and Plaintiff's Identity on Punitive Damage Awards, Law and Human Behavior, 1999, Vol. 23/4, S. 449 (463). Die empirischen Studien beziehen sich auf das amerikanische Jury-System. Allerdings sollten ihre Ergebnisse auf das deutsche Rechtssystem übertragbar sein. Es gibt keine Hinweise darauf, dass Richter Ankereffekten stärker widerstehen könnten als Mitglieder einer Jury. Dazu B. Englich/T. Mussweiler, Sentencing Under Uncertainty: Anchoring Effects in the Courtroom, Journal of Applied Social Psychology 2001, Vol. 31/7, S. 1535 (1536 f. m.w.N.).

118 B. Englich/T. Mussweiler, Sentencing (Fn. 118), S. 1535 (1537ff.).

119 B. Englich, Urteilseinflüsse (Fn. 77), S. 490 m.w.N.

120 B. Englich, „Geben Sie ihm doch einfach fünf Jahre!”, Zeitschrift für Sozialpsychologie 2005, S. 215.

121 B. Englich, Urteilseinflüsse (Fn. 77), S. 490 f. m.w.N.

122 T. Mussweiler/F. Strack, Comparing is believing: A selective accessibility model of judgmental anchoring, European Review of Social Psychology 1999, Vol. 10, S. 135 (158 m.w.N.).

123 Ähnlich B. Englich, Urteilseinflüsse (Fn. 77), S. 494 m.w.N. 
wissenschaftlich fundierte Überlegungen zu ersetzen und Gerichtsentscheidungen auf eine sachlichere Grundlage zu stellen.

\section{a) Alltagstheorien und Gerichtsgutachten}

Ein Sachverständiger ist verpflichtet, sein Gutachten nach den Regeln der wissenschaftlichen Kunst zu erstellen. Er muss die Qualitätsstandards beachten, die in seiner Wissenschaftsdisziplin gelten. ${ }^{124}$ Er hat die Untersuchungsmethoden und Theorien anzuwenden, die in seiner Wissenschaft akzeptiert sind. Dabei muss er in jeder Hinsicht den aktuellen Forschungsstand der Wissenschaft beachten. Auf dieser Grundlage soll er Ergebnisse finden, die wissenschaftlich fundiert sind. Das ist das Gegenteil von richterlichen Alltagstheorien, die auf persönlichen Erfahrungen, Kenntnissen, Werturteilen und Vorurteilen basieren. Auch wenn Gerichtsgutachten nicht selten fehlerhaft sind: ${ }^{125}$ Sie leisten einen wichtigen Beitrag zur Qualitätsverbesserung von Gerichtsentscheidungen. Denn sie ersetzen - das ist der theoretische Idealfall - die subjektiv gefärbten, nicht selten hoch emotionalen Alltagstheorien des Richters durch wissenschaftlich fundierte, nüchterne und objektivierbare Erklärungen. Allerdings darf eines nicht verkannt werden: Wenn Gutachten fehlerhaft sind, führen Sie zu fehlerhaften Gerichtsentscheidungen. Dann den Fehler im Urteil oder Beschluss $\mathrm{zu}$ entdecken, ist besonders schwierig. Denn er ist Bestandteil eines fachwissenschaftlichen Gutachtens und letztlich - wenn überhaupt - nur für Fachwissenschaftler sichtbar.

\section{b) Ankereffekt und Gerichtsgutachten}

Welchen Ankereffekt haben die Ergebnisse eines Gerichtsgutachtens? Dazu gibt es soweit ersichtlich - bisher keine empirischen Studien. Es spricht aber aus theoretischer Sicht vieles dafür, dass ein Gerichtsgutachten einen starken Ankereffekt auf das spätere Urteil des Gerichts hat. Ankereffekte treten sogar dann auf, wenn völlig sinnlose oder eindeutig parteiische Informationen gegeben werden. Dann sollten die unparteiischen, wissenschaftlich fundierten Informationen eines Experten erst recht diese Wirkung zeigen. Hinzu kommt: Informationen, die man selbst generiert, werden als weniger verzerrt wahrgenommen als Informationen, die von außen vorgegeben werden. ${ }^{126}$ Vor diesem Hintergrund dürften die Ankereffekte von Gutachten eher noch stärker sein. Denn der Richter ist ja an der Entstehung des Gutachtens mitbeteiligt - durch die Auswahl des Gutachters und die Formulierung des Gutachtenauftrags. Starke Ankereffekte eines Gutachtens sind eine wichtige, noch wenig untersuchte Ursache für den großen Einfluss von Sachverständigen auf die Urteile von Gerichten.

124 Grundlegend BGHSt 45, 164 (178 f.); BGH NStZ 2005, 205; NStZ 2004, 437; st. Rspr.

125 Zur Qualität von Schuldfähigkeitsgutachten sehr kritisch A. Schöttle, Die Schuldfähigkeitsbegutachtung in Jugendstrafverfahren - Eine Bestands- und Qualitätsanalyse, Berlin 2013, S. 65 ff. m. umfangr. Material; K. Schnoor, Beurteilung der Schuldfähigkeit, Köln 2009, S. 30 ff.

126 B. Englich, Urteilseinflüsse (Fn. 77), S. 492 m.w.N. 


\section{c) Fazit: Gerichtsgutachten als Chance und Risiko}

Gerichtsgutachten bergen große Chancen. Sie können die problematischen Alltagstheorien der Richter verdrängen. Im besten Falle schaffen Sie eine fachwissenschaftlich fundierte und objektive Sachgrundlage für eine Gerichtsentscheidung. Mit Gerichtsgutachten sind aber auch Risiken für die richterliche Tätigkeit verbunden. Wenn fehlerhafte Gutachten in ein Urteil eingeflossen sind, lässt sich das im Instanzenzug nur sehr schwer wieder rückgängig machen. Denn Fehler in Sachverständigengutachten lassen sich nur schwer identifizieren. ${ }^{127}$

\section{Die Rolle der Emotionen}

Emotionen steuern menschliches Verhalten sehr weit gehend. Welche Entscheidungen Menschen treffen, hängt zu einem großen Teil von ihren Gefühlen ab. Das gilt auch für die Entscheidungen von Richtern. Diese Erkenntnis kollidiert mit dem Selbstbild der Justiz als einer nüchternen, rationalen Instanz. Welche Rolle spielen wissenschaftliche Sachverständigengutachten in diesem Zusammenhang?

\section{Emotionen und Entscheidungen}

Emotionen haben einen großen Einfluss auf die Willensbildung. ${ }^{128}$ Sie beeinflussen das Urteilen und Schlussfolgern. ${ }^{129}$ Emotionen wirken sich auch auf die Informationsverarbeitung aus. Positive Affekte erweitern und öffnen den Aufmerksamkeitsfokus; negative zentrieren die Aufmerksamkeit dagegen auf wenige Inhalte und ausgesuchte Details. ${ }^{130}$ Auch die Gedächtnisleistung hängt zu einem erheblichen Teil von Emotionen ab. Starke Emotionen erleichtern es, neue Inhalte in das Gedächtnis einzuspeichern; genauso können Sie aber auch gegenteilige Effekte entwickeln. ${ }^{131}$ Mit anderen Worten: Emotionen sind ein überragend wichtiger Faktor, wenn Menschen entscheiden oder urteilen. Die Macht der Emotionen beeinflusst das menschliche Verhalten und den Willen des Menschen sehr stark. ${ }^{132}$ Was bedeutet das für richterliche Entscheidungen und Urteile?

127 Sehr instruktiv dazu die empirische Untersuchung von J. Fegert u.a., Bestandsaufnahme und Qualitätssicherung der forensisch-psychiatrischen Gutachtertätigkeit in Mecklenburg-Vorpommern bei Tötungs- und Brandstiftungsdelikten, Schwerin 2003, S. $89 \mathrm{ff}$.

128 Einzelheiten dazu bei G. Loewenstein/J. Lerner, The Role of Affect in Decision Making, in: R. Davidson u.a. (Hrsg.): Handbook of Affective Science, Oxford: OUP 2003, S. 3; P. Zimbardo/R. Gerrig, Psychologie (Fn. 89), S. 619 ff. m.w.N.

129 P. Zimbardo/R. Gerrig, Psychologie (Fn. 89), S. 465 f. m.w.N. Im Detail dazu J. Forgas, Affective Influences on Attitudes and Judgments, in: R. Davidson u.a. (Hrsg.): Handbook of Affective Science, Oxford: OUP 2003, S. 604 ff. m.w.N.

130 K. Rothermund/A. Eder, Allgemeine Psychologie: Motivation und Emotion, Berlin 2009, S. 682.

131 . G. Roth, Fühlen, Denken, Handeln. Wie das Gehirn unser Verhalten steuert, Frankfurt/Main 2003, S. 307 m.w.N.

132 L. Ciompi/E. Endert, Gefühle machen Geschichte: Die Wirkung kollektiver Emotionen - von Hitler bis Obama, Göttingen 2011, S. 20 f. m.w.N. sehen Emotionen sogar als entscheidende energetische Treiber menschlichen Verhaltens an. 


\section{Emotionen und Entscheidungen von Richtern}

Das moderne westliche Recht ignoriert die Bedeutung der Emotionen (fast) völlig. ${ }^{133}$ Juristische Kommunikation nimmt Rationalität für sich in Anspruch. ${ }^{134}$ Rechtsprobleme rational, ohne Emotionen zu lösen - das ist die Idealvorstellung des modernen westlichen Rechts. Seine Entwicklung wird nicht selten als Prozess einer permanenten, immer weiter zunehmenden Rationalisierung beschrieben. ${ }^{135}$ Hinter dieser Vorstellung lässt die alte Dichotomie von Rationalität und Emotionalität entdecken, die seit Platon im kulturellen Gedächtnis verankert ist und prägende Wirkungen auf das (Rechts)Denken entfaltet hat. Sie wertet Emotionen gegenüber der Rationalität $\mathrm{ab}$.

Vor diesem Hintergrund ist es wenig verwunderlich, dass Emotionen im Recht eher als Problem und Gefahr betrachtet werden. Juristen sehen sich als vernünftige und rationale Technokraten, die - sine ira et studio - Probleme lösen. ${ }^{136}$ Emotionen machen das - scheinbar - schwieriger. Nach der immer noch vorherrschenden Meinung unter Juristen sind Gefühle eher ein Problem, das vernünftige rationale Lösungen verhindert. Die Folge: Gefühle werden verdeckt, verleugnet und verdrängt. Gefühle lassen sich aber auf Dauer nicht verdrängen. Auch ignorierte Gefühle entfalten Wirkungen - allerdings völlig unkontrolliert. Das ist - nicht nur aber gerade auch - im Gerichtssaal ein Problem. Denn Gerichtsverfahren sollen von Vernunft und Rationalität geprägt sein, nicht von verdrängten Gefühlen. Welche Rolle spielen Gerichtsgutachten in diesem Zusammenhang?

\section{Emotionen und Sachverständigengutachten}

Sachverständigengutachten können ein Mittel sein, ein Gerichtsverfahren zu rationalisieren. Im Idealfall liefern Sachverständige fundierte, wissenschaftlich begründete objektive Fakten. Das verringert den Spielraum für richterliche Entscheidungen, die von verdrängten Emotionen geprägt sein könnten. Das lässt sich am Beispiel der richterlichen Alltagstheorien zeigen. Wie jeder Mensch, erklären sich Richter die Welt auch mit Hilfe so genannter Alltagstheorien. ${ }^{137}$ Alltagstheorien entstehen aus einer Mischung von Lebenserfahrung, Wertvorstellungen, Gefühlen und Vorurtei-

133 K. Abrams/H. Keren, Who's Afraid of Law and the Emotions? Minnesota Law Review 2009, S. 1997 (2003) m.w.N. Dazu M. Weber, Wirtschaft und Gesellschaft - Grundriß der verstehenden Soziologie, Frankfurt a.M. (1972/1921), S. 563 ff., der auch Rechtsordnungen schildert, in denen das anders ist (war).

134 Für die herrschende Meinung H. Schulze-Fielitz, Leistungsgrenzen des Verfassungsrechts, Berlin 2003, S. 444 f. m.w.N., der die „strikt juridische Rationalität“ vor allem auf die „abstrahierende bilderlose Sprache von geschriebenen Texten “ zurückführt.

135 So stellt M. Weber, Wirtschaft (Fn. 134), S. 395 f. die Entwicklung des modernen Rechts dar.

136 Zum Hintergrund M. Weber, Wirtschaft (Fn. 134), S. 561 ff., der das in einen Zusammenhang mit der Entwicklung der modernen Wirtschaft und der Bürokratie bringt.

137 Ausführlich dazu J. Urteilseinflüsse (Fn. 103), Richterliche Alltagstheorien im Bereich des Zivilrechts. Tübingen 1984, S. 65 ff. 
len. ${ }^{138}$ Sie sind letztlich stark emotional geprägte Theorien, um Geschehnisse des Alltags zu erklären. Sie finden sich auch in Hülle und Fülle in unterschiedlichen Urteilen und Beschlüssen. Sie führen aber dazu, dass die Rechtsprechung in Teilen emotionalisiert wird und irrational entscheidet. Sachverständigengutachten sind im Idealfall völlig anders - nämlich wissenschaftlich fundiert, nüchtern, sachlich objektiv und nicht emotional. Urteile, die auf Gerichtsgutachten statt auf Alltagstheorien fußen, sind letztlich rationaler, objektiver und gerechter.

Das ist der theoretische Idealfall. Die Realität ist komplexer. Denn Sachverständigengutachten sind nicht immer so objektiv, wie sie sein sollten. Der Grund dafür liegt auf der Hand: Auch Sachverständige sind Menschen, die von Emotionen gesteuert werden. Auch Sie sind nicht davor gefeit, Stimmungen, Werturteile und Vorurteile in ihr Gutachten einfließen zu lassen. Im schlimmsten Fall führt ein missglücktes Gutachten dann dazu, dass ein Gerichtsverfahren - unter dem Mantel der Wissenschaftlichkeit - weiter emotionalisiert und irrationalisiert wird

\section{Die Suche nach Bestätigung - Der confirmation bias im Gerichtssaal}

Die menschliche Wahrnehmung ist selektiv. Im Gerichtssaal ist das problematisch. Denn dort geht es darum, alle relevanten Fakten zu finden und in einer Entscheidung zu verarbeiten. Gutachten können ein Mittel sein, der selektiven Wahrnehmung von Richtern entgegenzuwirken. Sicher ist das aber nicht.

\section{Der confirmation bias}

Die menschliche Wahrnehmung ist vom confirmation bias ${ }^{139}$ geprägt. Menschen nehmen nicht unvoreingenommen und objektiv, sondern selektiv wahr. Sie nehmen Informationen, die ihre Meinung und Weltsicht bestätigen, eher zur Kenntnis und messen ihnen ein höheres Gewicht bei. ${ }^{140}$ Informationen, die sie zu einer Meinungsänderung veranlassen könnten - oder müssten - werden dagegen kaum registriert und nur schwach gewichtet. Etwas zugespitzt lässt sich sagen: Menschen suchen nach Informationen, die ihre Meinungen und (Vor)Urteile bestätigen. Andere Informationen nehmen sie nur ungerne oder gar nicht zur Kenntnis. Über die Ursachen dieses - schon lange bekannten und früh beschriebenen ${ }^{141}$ - Phänomens herrscht noch Un-

138 Urteilseinflüsse (Fn. 103)J. Urteilseinflüsse (Fn. 103), Richterliche Alltagstheorien im Bereich des Zivilrechts. Tübingen 1984, S. 69 ff. m.w.N. Ausführlich dazu s.o. D.II.2.

139 Zum Begriff J. Klayman, Varieties of Confirmation Bias, in: J. Busemeyer u.a.(Hrsg.), Decision Making from a Cognitive Perspective, London: Academic Press 1995, S. 385.

140 R. Nickerson, Confirmation Bias, Review of General Psychology 1998, S. 175 (177 ff. m.w.N.).

141 R. Nickerson, Bias (Fn. 142), S. 175 (176), zeigt, dass Francis Bacon dieses Phänomen schon 1620 beschrieben hat. 
klarheit. ${ }^{142}$ An seiner Existenz besteht aber - wie vielfältige empirische Daten belegen - kein Zweifel. ${ }^{143}$

\section{Confirmation bias und Gerichtsgutachten}

Richter sollen alle Fakten eines Falles unvoreingenommen und objektiv recherchieren, rezipieren und bewerten. Das ist eine fast utopische Forderung. Denn die Informationsverarbeitungskapazität des Menschen ist begrenzt. ${ }^{144}$ Der confirmation bias steuert den notwendigen kognitiven Filtervorgang: ${ }^{145}$ Aus der Informationsflut werden die Informationen herausgesucht, wahrgenommen und rezipiert, die zur bereits vorhandenen Meinung passen. Alle anderen werden (fast) ignoriert. Das birgt die Gefahr, dass Informationen, die für eine Gerichtsentscheidung wichtig sind, im Verfahren nicht beachtet werden.

Welche Rolle spielen in diesem Zusammenhang die gerichtlichen Sachverständigengutachten?

Ein Gerichtsgutachten erweitert im Idealfall den Horizont des Gerichts deutlich. Es wird mit fachlichen Informationen versorgt, die es vorher nicht hatte. Wenn die Richter sich auf die neuen, vielleicht sogar völlig überraschenden Informationen einlassen, wirken sie dem confirmation bias entgegen. Ganz sicher ist das nicht. Denkbar ist auch ein anderer Fall: Das Gericht rezipiert aus dem Gutachten nur die Fakten und Informationen, die es erwartet. Dann wird das Gutachten entwertet - durch die selektive Wahrnehmung der Richter. Ebenso problematisch ist eine andere, nicht seltene Konstellation: Der Gutachter spürt die Erwartungen des Gerichts und bedient sie in seinem Gutachten. ${ }^{146}$ Auch in diesem Fall wird die Chance vertan, den confirmation bias mit Hilfe des Sachverständigengutachtens zu überwinden.

\section{E. Der Sachverständige als Gehilfe des Gerichts}

Der Gerichtsgutachter darf den Richtern nicht durch seine Sachkunde dominieren. Genauso fatal ist aber der gegenteilige Fall. Ein Sachverständiger darf sich nicht zum willfährigen Gehilfen des Gerichts machen. Auch dann verletzt er seine Rolle als sachkundiger, objektiver Berater der Justiz. In der komplexen Balance zwischen Sachverständigen und Richtern muss auch diese problematische Rollenverletzung vermieden werden. Wie lässt sich das erreichen?

142 Detailliert zu den unterschiedlichen Erklärungsansätzen R. Nickerson, Bias (Fn. 142), S. 175 (197ff. m.w.N.). Eine eigene, plausible These stellt J. Klayman, Varieties (Fn. 141), S. 411 auf.

143 J. Klayman, Varieties (Fn. 140), S. 406. Einen umfassenden Überblick über die empirischen Studien gibt R. Nickerson, Bias (Fn. 141), S. 175 (177 ff. m.w.N.).

144 P. Zimbardo/R. Gerrig, Psychologie, 16. Aufl. München 2004, S. 171 f. m.w.N.

145 Möglicherweise ist die Filterfunktion sogar die Ursache für die Entstehung des confirmation bias in der menschlichen Entwicklungsgeschichte. Zu diesen Theorien R. Nickerson, Bias (Fn. 142), S. 175 (198 f. m.w.N.).

146 Ausführlich dazu s.u. E. 


\section{Engel der Medizin - oder Reserveengel der Jurisprudenz?}

Ein Problem ist in der Praxis nicht selten: Der Gutachter verlässt seine Rolle als unparteiischer, wissenschaftlicher Informationsdienstleister und wird zum Ermittlungsgehilfen des Gerichts. ${ }^{147}$ Er wird - wie es Robert Musil in seinem „Mann ohne Eigenschaften“ ausgedrückt hat - vom Engel der Medizin zum Reserveengel der Jurisprudenz. ${ }^{148}$ Damit verletzt er seine Pflicht zur Neutralität und zur Objektivität. Wie kann es zu diesem Rollenwechsel kommen?

\section{1. Überzeugungen, Werte, Vorurteile des Gutachters}

Wie alle anderen Menschen auch haben Gutachter eigene Überzeugungen, Grundwerte und Lebensanschauungen. Selbstverständlich beeinflussen sie das Denken und Handeln der Gutachter. ${ }^{149}$ Persönliche Sichtweisen und Vorurteile können einen Gutachter beeinflussen. ${ }^{150}$ Oder er identifiziert sich mit der Justiz und schlüpft in die Rolle des Staatsanwalts. ${ }^{151}$ Dann verliert das Gutachten seinen wissenschaftlichen Wert, der in der Objektivität ${ }^{152}$ und Neutralität ${ }^{153}$ liegt. Gerade in hoch emotionalen Bereichen kommt es vor, dass Gutachter die Rolle wechseln - getrieben von Grundüberzeugungen oder Vorurteilen. ${ }^{154}$ Ein Beispiel dafür ist das Sexualstrafrecht, in dem „Verdammungsurteile“ statt objektiver Gutachten nicht selten sind. ${ }^{155}$

Die Gefahr des Rollenwechsels ist nicht in allen Wissenschaftsdisziplinen gleich groß. Sie ist eher groß bei den Menschenwissenschaften wie Medizin, Psychiatrie, Psychologie oder Pädagogik. Hier geht es - etwa im Familienrecht oder im Strafrecht - um emotionale Sachverhalte. Deshalb besteht die Gefahr, dass auch Sachverständige da-

147 H. Kury/J. Obergfell-Fuchs (Fn. 3), S. 106 m.w.N.; N. Nedopil/J. L. Müller, Psychiatrie (Fn. 2), S. 425 m.w.N. Schon früher G. Heinz, Fehlerquellen (Fn. 35), S. 59 ff.

148 R. Musil, Der Mann ohne Eigenschaften, Berlin 1994, Kap. 60. a.E.

149 Grundsätzlich zum komplexen, nicht linearen Einfluss von Einstellungen auf das menschliche Verhalten P. Zimbardo/R. Gerrig, Psychologie (Fn. 89), S. 644 ff. m.w.N.

150 H. Kury/J. Obergfell-Fuchs (Fn. 3), S. 106 m.w.N.; G. Heinz, Fehlerquellen (Fn. 35), S. 100 f. .

151 H. Kury/J. Obergfell-Fuchs (Fn. 3), S. 112. Ausführlich dazu auf empirischer Grundlage G. Heinz, Fehlerquellen (Fn. 35), S. 56 ff.

152 Anders als der Laie meint, sind nicht alle Wissenschaften objektiv. In der klinischen Medizin und in medizinischen Gutachten lässt sich eine absolut objektive und vollständig zutreffende Diagnose in der Regel nicht erreichen. Dazu F. Anschütz, Zur Objektivität der Befunderhebung und der Diagnose im ärztlichen Gutachten, in: K. Brückner/G. Dalichau (Hrsg.), Beiträge zum Sozialrecht. Festgabe für Hans Grüner, Starnberg 1982, S. 31. Wie Gerichte im Idealfall damit umgehen können skizziert K. Brückner, Zur Objektivität des medizinischen Sachverständigengutachtens, in: K. Brückner/G. Dalichau (Hrsg.), Beiträge zum Sozialrecht. Festgabe für Hans Grüner, Starnberg 1982, S. 100 ff.

153 Zur Neutralität des technischen Sachverständigen aus rechtsvergleichender Perspektive F. Nicklisch, Generalbericht, in: F. Nicklisch (Hrsg.), Der Technische Sachverständige im Prozess, Heidelberg 1984, S. 245 ff. m.w.N.

154 Dazu N. Konrad/W. Rasch, Psychiatrie (Fn. 42), S. 24 m.w.N.

155 N. Nedopil/J. L. Müller, Psychiatrie (Fn. 2), S. 425 m.w.N.; I. Rode/A. Legnaro, Sachverständige (Fn. 3), S. 19, die, a.a.O., S. 11, eine psychoanalytisch gefärbte Erklärung für diese Überidentifikation bieten. H. Kury/J. Obergfell-Fuchs (Fn. 3), S. 107 sind aber der Ansicht, dass die Zahl solcher Gutachten inzwischen deutlich zurückgegangen ist. 
rauf mit Emotionen reagieren. ${ }^{156}$ Völlig anders ist die Problemlage bei naturwissenschaftlichen oder technischen Sachverständigen. Die Fragen, die sie in ihren Gutachten beantworten sollen, sind in der Regel nüchtern und nicht emotional. Dementsprechend gering ist die Versuchung, im Gutachten eigene Emotionen zu verarbeiten, die den wissenschaftlichen Blick trüben könnten.

\section{2. Ökonomische Abhängigkeiten}

Manchmal ist der Grund für den Rollenwechsel des Gutachters ganz profan ökonomisch: Er ist finanziell auf die Aufträge des Gerichts angewiesen. Finanzielle Abhängigkeiten sind nicht selten. Nach einer neueren empirischen Studie erzielen knapp $30 \%$ der psychiatrischen Gutachter über $50 \%$ ihrer Gesamteinnahmen aus Gerichtsgutachten. ${ }^{157}$ Bei den psychologischen Sachverständigen ist die Abhängigkeit noch ausgeprägter: Fast die Hälfte bestreitet über $50 \%$ ihres Einkommens aus der Gutachtertätigkeit. ${ }^{158}$

Diese Abhängigkeit hat fatale Folgen. Der Gutachter will - vielleicht sogar: muss sich Folgeaufträge sichern. ${ }^{159}$ Das geht letztlich nur, wenn er - wie auch immer - die Erwartungen des Gerichts erfüllt. Dieses Kalkül geht oft auf. Gerichte neigen dazu, verstärkt Gutachter zu bestellen, mit denen sich eine angenehme Zusammenarbeit ohne Reibungen und Überraschungen eingespielt hat. ${ }^{160}$

\section{Erwartungshaltung des Gerichts}

Eine wichtige Ursache für den Rollenwechsel, den mancher Gutachter vollzieht, ist die Erwartungshaltung des Gerichts, die es dem Gutachter kommuniziert.

Welche Erwartungen ein Gericht hat, zeigt sich schon darin, welche Gutachter es bestellt. ${ }^{161}$ Ist es eine fachliche Koryphäe, die für eigenständige Gedanken und Gutachten bekannt ist? Oder ist es eine Person, die im Ruf steht, kurze und schnelle also unter prozessökonomischen Gesichtspunkten unproblematische - Gutachten anzufertigen, die Erwartungen des Richters bestätigen? Wird ein konservativer Gutachter bestellt, der das Weltbild konservativer Richter teilt? Oder bekommt ein innovativer Fachmann den Gutachtenauftrag, der traditionelle Denkgewohnheiten und Weltanschauungen in Frage stellt?

156 Emotionen sind „ansteckend“. Dazu grundlegend E. Hatfield u.a., Emotional Contagion, Cambrige: Cambridge University Press 1993, S. 48 ff. und pass.

157 B. Jordan/U. Gresser, Gutachter (Fn. 9), S. 71 (74).

158 B. Jordan/U. Gresser, Gutachter (Fn. 9), S. 71 (74).

159 N. Nedopil/J. L. Müller, Psychiatrie (Fn. 2), S. 425.

160 Zur „Hausgutachterproblematik“ H. Kury/J. Obergfell-Fuchs (Fn. 3), S. 107 m.w.N.; G. Tondorf/B. Tondorf, Sachverständige (Fn. 43), Rn. 244 f. m.w.N. und S. Barton, Sachverständiger und Verteidiger, StV 1983, S. 73 (74) auf der Basis einer empirischen Untersuchung.

161 Dazu N. Konrad/W. Rasch, Psychiatrie (Fn. 42), S. 199. 
Es wird eher selten vorkommen, dass ein Richter einem Gutachter expressis verbis seine Erwartungen mitteilt. Aber wer als Gutachter zwischen den Zeilen lesen kann, wird keine Schwierigkeiten haben, die Erwartungshaltung des Gerichts herauszulesen. Eine neuere empirische Studie zeigt, dass Gerichte ihren Gutachtern durchaus signalisieren, welche Tendenz ein Gutachten haben sollte. Fast jeder vierte medizinisch-psychologische Gutachter gibt an, dass ihm ein Richter gelegentlich oder sogar häufig signalisiert habe, welche Tendenz das Gutachten haben solle. ${ }^{162}$

Der Richter signalisiert dem Gutachter, was er von ihm erwartet. Der Gutachter erfüllt die Erwartungen. Deshalb wird er bald wieder mit einem Gutachten beauftragt. Auf dieser Basis entwickeln sich enge, sich selbst stetig verstärkende Beziehungen zwischen Gerichten und Gutachter. Dass es diese „Hausgutachter-Problematik“ gibt, lässt sich auch mit Zahlen belegen. ${ }^{163}$

\section{Rollenbewusstsein und persönliche Souveränität}

Der Gutachter als willfähriger Gehilfe des Gerichts? Natürlich entspricht diese Konstellation ebenfalls nicht der idealtypischen Beziehung zwischen Richter und Gutachter, die das Verfassungsrecht vorschreibt. Der Gutachter soll objektive und neutrale Informationen liefern, die das Urteil auf eine wissenschaftlich fundierte Basis stellen. Das gelingt nicht, wenn er mit seinem Gutachten nur das richterliche VorUrteil bestätigt.

Wie lassen sich solche Konstellationen vermeiden? Beide Seiten - Richter und Gutachter - müssen sich ihrer Rolle bewusst sein und ihre Grenzen akzeptieren. Die Rolle des Richters ist, in intellektueller Offenheit die fachliche Hilfe des Gutachters anzunehmen. Er darf ihn nicht dazu missbrauchen, die richterlichen Vor-Urteile durch wissenschaftlich verbrämte Gutachten zu bestätigen. Die Rolle des Gutachters ist die des wissenschaftlichen Beraters. Er muss das Gericht durch seinen fachlichen Sachverstand unterstützen. Dazu gehört auch, Erwartungen der Richter zu enttäuschen, wenn der wissenschaftlichen Forschungsstand das erfordert.

Neben diesem Bewusstsein für ihre Rolle im Prozess müssen beide Seiten persönliche Souveränität aufbringen. Der Richter muss akzeptieren, dass ihm vom Gutachter widersprochen wird und seine Vor-Überzeugungen ins Wanken geraten können. Der Gutachter muss sich bewusst sein, dass es beim Gutachten um einen Beitrag zur

162 B. Jordan/U. Gresser, Gutachter (Fn. 9), S. 71 , 76. Wie die Zahlen belegen, wird Gutachtern, die wirtschaftlich abhängig von Gerichtsgutachten sind, sogar noch deutlich häufiger eine Tendenz signalisiert. Etwas andere Zahlen finden sich in einer älteren Studie: A. Böttger u.a., Richter in Weiß oder Gehilfe des Gerichts? MSchrKrim 1991, S. 369 (376). Hier geben etwa 11 \% der Gutachter an, häufig oder fast immer von Richtern im Hinblick auf ein gewünschtes Ergebnis beeinflusst zu werden.

163 Dazu auch H. Kury/J. Obergfell-Fuchs (Fn. 3), S. 107. Schon früher auf empirischer Grundlage K. Schnoor, Beurteilung der Schuldfähigkeit, Köln 2009, S. 115 f. und V. Dittmann u.a., Erfahrungen von Juristen mit forensisch-psychiatrischen Sachverständigen, Forensia 9, 1988, S. 219 (226). 
Wahrheitsfindung geht. Ein gerichtliches Sachverständigengutachten ist kein zulässiges Mittel, Beziehungen zu einem Richter zu pflegen, um Folgeaufträge zu sichern.

\section{F. Der Richter und sein Berater}

Die Machtbalance zwischen Richter und Gutachter ist schwierig. Die faktische Macht der Gutachter ist groß; die potentielle Ohnmacht der Richter ist beunruhigend. Das ist letztlich auch ein verfassungsrechtliches Problem. Denn Art. 92 GG ist eindeutig: Der Richter, nicht der Gutachter spricht das Urteil. Welche Lösungsansätze gibt es, um das heikle Verhältnis immer wieder in der verfassungsrechtlich gebotenen Balance zu halten?

\section{Psychologische Grundlage: Selbstreflexion und Bewusstsein statt Abwehr}

Die Macht der Gutachter wird in der Justiz weit gehend verdrängt. Über die Strukturen und Mechanismen, die den Einfluss der Gutachter ausmachen, wird wenig gesprochen. Dieses psychisch bedingte Abwehrverhalten hilft nicht weiter. Verdrängung macht hilflos. Man ist dem, was man verdrängt, schutzlos ausgeliefert. Für die Justiz hat das eine Konsequenz: Richterinnen und Richter müssen sich immer der potentiellen Macht der Gutachter bewusst sein. Nur dann können sie mit ihr kritisch und konstruktiv umgehen. Verdrängen Sie die Problematik, die mit dem Gutachterwesen verbunden ist, sind sie dem Einfluss der Gutachter hilflos ausgeliefert. Genau so wichtig ist, die sozialpsychologischen und emotionalen Implikationen der Beziehung zwischen Richter und Gutachter zu reflektieren. Dann besteht die Chance, dass der Richter „Herr im eigenen Haus“164 ist - und nicht seine Emotionen, Vorurteile und Alltagstheorien.

\section{Ausserrechtliche Fachkompetenz : Sachverständige auf der Richterbank?}

Die Übermacht der Sachverständigen beruht - nicht nur, aber auch - auf der mangelnden Fach - und Sachkunde der Juristen. ${ }^{165}$ Ein Mittel, den Einfluss der Sachverständigen zu relativieren, ist also eine stärkere außerrechtliche Fachkompetenz der Juristen. Das lässt sich durch organisatorische Maßnahmen und durch individuelle Fortbildung erreichen.

\section{Kompetenz durch Gerichtsorganisation}

Gerichte kommen nicht ohne Gutachter aus, die ihnen das notwendige fachliche Know-how zur Verfügung stellen. Trotzdem brauchen Richter ein Mindestmaß an eigener Fachkompetenz in dem ihnen an sich fremden Fachgebiet. Ohne Experten zu

164 S. Freud, Eine Schwierigkeit der Psychoanalyse, in: Imago, Bd. V 1917, S. 7, spricht davon, dass das „Ich“ nicht „Herr sei im eigenen Haus“.

165 A. Kaufmann, Problem (Fn. 39), S. 1071. 
sein, müssen Sie einen geeigneten Gutachter finden können. ${ }^{166}$ Sie müssen das Gutachten dann in den Grundzügen verstehen und wenigstens unter Plausibilitätsaspekten kritisch hinterfragen können. ${ }^{167}$ Nur dann erfüllen Sie Ihre Pflicht, auch in schwierigen Fachfragen ein eigenes Urteil zu sprechen. ${ }^{168}$ Das geht nur, wenn die Richter über längere Erfahrungen mit Problemstellungen aus einem Fachgebiet verfügen und sich mindestens Grundkenntnisse angeeignet haben. ${ }^{169}$ Die Richter befinden sich hier in einem Dilemma. ${ }^{170}$ Weil ihnen Fachkenntnisse fehlen, müssen Sie einen Gutachter beauftragen. Um das Gutachten aber wirklich verstehen zu können, müssten sie Fachkenntnisse haben.

Vor allem Bundesgerichte stellen durch Organisationsmaßnahmen eine Spezialisierung - und damit Fortbildung - ihrer Richter sicher. ${ }^{171}$ Spezialisierte Senate und Kammern ermöglichen eine doppelte Fortbildung ihrer Richter. Die Richter erwerben Spezialkenntnisse nicht nur über das spezielle Rechtsgebiet, sondern auch über das technische oder naturwissenschaftliche Sachgebiet. Einen guten Weg schlägt der Gesetzgeber mit $\mathbb{3} 348$ ZPO ein. Die Norm sieht vor, dass in medizinrechtlichen Prozessen kein Einzelrichter, sondern eine Spezialkammer entscheidet. Dadurch ist sichergestellt, dass die Richter über medizinische Grundkenntnisse verfügen. Das sollte sie in die Lage versetzen, medizinische Gutachten zu verstehen und kritisch zu rezipieren. Das könnte - und ist es faktisch auch schon - ein Modell für die Bewältigung sachlich-fachlich komplizierter Fälle sein.

\section{Fachkompetenz durch zertifizierte Fachrichter}

Allerdings reicht dieses richterliche training on the job nicht aus. Es dauert relativ lange und ist zu punktuell und unsystematisch. Letztlich kann es deshalb mit dem fachwissenschaftlichen Fortschritt nicht Schritt halten.

In vielen - auch juristischen - Berufen lassen sich zunehmende Spezialisierungen beobachten. Das hängt mit der explosionsartigen Zunahme und der extremen Ausdifferenzierung des Wissens zusammen. Nur Spezialisten können noch die Details mancher Problematik überblicken. Dass etwa die Zahl der Fachanwälte in den letzten

166 Zur Problematik der Gutachterauswahl H. Kury/J. Obergfell-Fuchs (Fn. 3), S. 89 ff. m.w.N. und K. Detter, Der Sachverständige im Strafverfahren, NStZ 1998, S. 57 (59 m.w.N.). Abschreckende Beispiele bei der Bestellung medizinischer Gutachter schildert H. Lanz, Zweiklassenrecht durch Gutachterkauf, ZRP 1998, S. 337 (338 f. m.w.N.).

167 Dass durchaus auch Juristen Gutachten aus anderen Fachgebieten auf ihre Qualität prüfen können, betont H. Kury, Zur Qualität forensischer Begutachtung, Praxis der Rechtspsychologie, 1999, S. 126 (134).

168 Dazu noch immer treffend BGHSt 8, 113 (118f.).

169 W. Sass, Der Sachverständige - weiterhin ein prozessuales Problemfeld, DS 2007, S. 256 (262) plädiert für ein „belastbares Halbwissen“. Strikt dagegen $S$. Bro $\beta$, Richter und Sachverständiger, dargestellt anhand ausgewählter Probleme des Zivilprozesses, ZZP 1989 Vol. 102, S. 413 (418f.).

170 Ähnlich K. Oebler, Zur Problematik der Sachverständigenauswahl, ZRP 1999, S. 285 (286).

171 H. Sendler, Richter und Sachverständige, NJW 1986, S. 2907 (2910) mit Beispielen. 
Jahrzehnten zugenommen hat, ${ }^{172}$ passt in diesen Trend. Das könnte eine Anregung für die Justiz sein. Wenn sie spezialisierte Fachrichter ausbildet und zertifiziert, wird die Justiz in der Lage sein, auch hochspezialisierte, komplexe Probleme der Wissensgesellschaft adäquat zu bewältigen. Zertifizierte Fachrichter sollten in der Lage sein, dem interdisziplinären Dialog mit dem Fachgutachter adäquat zu führen. ${ }^{173}$ Um es zuzuspitzen: Die juristische Kritik an einem Gutachten darf nicht nur Ausdruck fachlicher Ignoranz sein. ${ }^{174}$

Diese Idee ist nicht neu. In manchen Bereichen ist der Einsatz von spezialisierten Fachrichtern vom Gesetzgeber bereits geregelt. Das Paradebeispiel dafür ist das Bundespatentgericht. Die Spruchkörper sind dort gemäß \67 PatG mit rechtskundigen und mit technischen Mitgliedern besetzt. Ein anderes Beispiel: $\mathbb{} 139$ Abs. 2 FlurbG etwa schreibt auch für die Flurbereinigungsgerichte eine Mischbesetzung vor. Neben hauptamtlichen Richtern gehören $\mathrm{zu}$ den Flurbereinigungssenaten ehrenamtliche Richter, die praktische Erfahrungen in Flurbereinigungsbehörden haben. Dasselbe Modell findet sich bei den Kammern für Handelssachen ( $\mathbb{S} 105 \mathrm{GVG}$ ). Sie sind mit hauptamtlichen Richtern und Handelsrichtern besetzt, die aus der wirtschaftlichen Praxis kommen und das notwendige ökonomische Fachwissen in den Prozess einbringen können.

\section{Doppelte Weichenstellung: Auswahl und Auftrag der Gutachter}

Die Entscheidung, dass ein Sachverständiger beauftragt wird, prägt den weiteren Verlauf des Prozesses ebenso wie die Entscheidung, welcher Gutachter konkret in die Pflicht genommen wird. Wenn diese grundlegenden Fragen beantwortet sind, gibt es noch eine weitere Weichenstellung, die nicht unterschätzt werden darf. Die konkrete Formulierung des Gutachtenauftrags an den Sachverständigen ist eine wichtige Voraussetzung dafür, dass ein qualitativ hochwertiges und für den Prozess hilfreiches Sachverständigengutachtens abgeliefert wird. ${ }^{175}$

\section{Das Gutachten}

Ob ein Gutachtenauftrag vergeben wird entscheidet das Gericht ganz allein. Hier werden Weichen für den weiteren Verlauf des Prozesses gestellt. Wenn es einen Gutachtenauftrag vergibt, akzeptiert ein Gericht seine fachlichen Grenzen. Es entscheidet sich dafür, unklare Sachfragen nicht mit Hilfe eigener Alltagstheorien, sondern wissenschaftlich fundiert zu klären. Das ist ein souveräner Verzicht auf Macht im Ge-

172 Siehe dazu die Statistik der BRAK unter http:/www.brak.de/w/files/04_fuer_journalisten/statistiken/ 2013/03_entwicklungfaebis 2013_.pdf.(8.8.2013).

173 So auch B. Voth, 30 Jahre Gerichtsgutachter - ein Erfahrungsbericht, BauR 1988, S. 666 (677) für den Bereich des Technikrechts. Ebenso für den psychiatrisch-psychologischen Bereich N. Konrad/W. Rasch, Psychiatrie (Fn. 42), S. 206 f. Strikt ablehnend aber H. Sendler, Richter und Sachverständige, NJW 1986, S. 2907 (2914 f. m.w.N.). Skeptisch auch H. Franzki, Diener (Fn. 4), S. 314 (316).

174 W. Rasch/N. Konrad, Forensische Psychiatrie, 3. Aufl. Stuttgart 2004, S. 38.

175 Grundsätzlich dazu H. Franzki, Diener (Fn. 4), S. 314 (318 f.). 
richtssaal. Gleichzeitig beginnt damit ein schwieriger Balanceakt für die Richter: Sie müssen die Fachkompetenz des Gutachters nutzen, ohne ihre richterliche Entscheidungsaufgabe an den Gutachter abzutreten. Die Versuchung, das zu tun, ist allerdings groß. ${ }^{176}$

\section{Der Gutachter}

Eine genauso wichtige Weichenstellung für den Prozess ist die Auswahl des Gutachters durch das Gericht. ${ }^{177}$ Die Richter haben dabei ein weites, aber - im Rechtsstaat selbstverständlich - kein unbegrenztes Auswahlermessen. Ein Gutachter muss zunächst fachlich kompetent und persönlich geeignet sein. ${ }^{178}$ Weil sich die Wissenschaften immer stärker spezialisieren und ausdifferenzierten, kann es im Einzelfall schwierig sein, einen solchen zu finden. ${ }^{179}$ Das Gericht darf hier allerdings keine Fehler machen. Sonst schafft es möglicherweise Gründe für eine Revision. ${ }^{180}$

Genauso wichtig sind andere, weniger fachliche als persönliche Eigenschaften, die ein Gerichtsgutachter im Idealfall hat. Er muss natürlich objektiv sein. Genauso wie ein Richter darf er nicht befangen sein. ${ }^{181}$ Dabei spielen auch die Grundeinstellungen eines Gutachters eine große Rolle. ${ }^{182}$ Er muss zum interdisziplinären Dialog mit dem Gericht bereit sein - und die Fähigkeit dazu haben. Er muss die innere Souveränität aufbringen, sich auf seine Rolle als Berater des Gerichts beschränken zu können. Nur wenn diese zusätzlichen Anforderungen erfüllt werden, kann der Gutachter spannungsfrei und sachlich zur Erforschung der Wahrheit beitragen.

\section{Das Wettbewerbsmodell}

Wie ließe sich die Abhängigkeit des Richters vom einzelnen Gutachter reduzieren? Eine Methode liegt auf der Hand: Jeder Gutachtenauftrag müsste parallel an zwei Gutachter vergeben werden, die sich nicht kennen und keinen Kontakt miteinander haben. Der Vergleich der beiden unabhängigen Gutachten würde den Richter in die Lage versetzen, jedes Gutachten mithilfe des jeweiligen anderen kritisch zu hinterfragen. Mit anderen Worten: Beide Gutachter treten unabhängig voneinander gegeneinander an. Aus dem Ergebnis dieses Wettbewerbs zieht der Richter dann seine Schlüsse. So gut dieses Verfahren funktionieren könnte: Die beschränkten finanziellen Mittel im Bereich der Justiz lassen eine Realisierung unrealistisch erscheinen.

176 Siehe dazu oben D.III.

177 H. Franzki, Diener (Fn. 4), S. 314 (317 f.) betont ebenfalls die Bedeutung der Gutachterauswahl. Zur Gutachterauswahl auf empirischer Grundlage V. Dittmann u.a., Erfahrungen von Juristen mit forensisch-psychiatrischen Sachverständigen, Forensia 9, 1988, S. 219 (225 f.).

178 H. Kater, Gutachten (Fn. 24), S. 60 f. zur Sachkunde ärztlicher Gutachter; F. Nicklisch, Generalbericht (Fn. 154), S. 240 zur fachlichen Kompetenz technischer Gutachter.

179 G. Wannagat, Sachverständige (Fn. 47), S. 349 (350).

180 Ähnlich H. Kater, Gutachten (Fn. 24), S. 59 m.w.N.

181 Dazu H. Franzki, Diener (Fn. 4), S. 314 (317f.).

182 R. Lamprecht, Außerrechtliche Einflüsse auf die richterliche Entscheidungsfindung, DRiZ 1989, S. 4 (5 m.w.N). 


\section{Der Gutachtenauftrag}

Die Formulierung des Gutachtenauftrags kann in ihrer Bedeutung kaum überschätzt werden. Die Formulierung des Beweisthemas muss so eindeutig erfolgen, dass Inhalt und Umfang des Gutachtenauftrags klar sind. ${ }^{183}$ Durch die Beweisfragen werden das Beweisthema und der Gutachtenauftrag dann im Einzelnen konkretisiert. Die im Prozess festgestellten (Anknüpfungs-) Tatsachen müssen dem Gutachter eindeutig mitgeteilt werden. ${ }^{184}$ Sie müssen in das Gutachten einbezogen werden. ${ }^{185}$ Zusätzliche Tatsachen dürfen dagegen nicht einfach im Gutachten berücksichtigt werden.

Hier liegt eine wichtige potentielle Ursache für Fehler des Gutachtens. Unklare Formulierungen des Gutachtenauftrags oder zu unkonkrete oder unvollständige Vorgaben können dazu führen, dass das Gutachten fehlerhaft wird oder für den Zweck des Prozesses unbrauchbar ist. ${ }^{186}$

Wie der Gutachtenauftrag formuliert wird und welche Fragen dem Gutachter gestellt werden, ist darüber hinaus von großer Bedeutung für die Machtverteilung im Prozess. Durch den konkreten Gutachtenauftrag entscheidet der Richter nolens volens über den Einfluss, den er dem Gutachter einräumt. Die Mischung von Sach-und Bewertungsfragen, ${ }^{187}$ die dem Sachverständigen vorgelegt werden, ist der entscheidende Punkt. Beschränkt sich der Gutachtenauftrag auf Sachfragen, hat der Sachverständige verhältnismäßig wenig Beurteilungsspielräume und Wertungsmöglichkeiten. Bewertungsfragen im Gutachtenauftrag dagegen erweitern die normativen Möglichkeiten des Gutachters. Die Folge: Sein Einfluss auf das Urteil wird größer, als er ohnehin schon ist.

Das lässt sich an einem Beispiel verdeutlichen. ${ }^{188}$ Die Frage: „Erlitt der Kläger nach dem Unfall einen Oberschenkelbruch?“ ist eine reine Sachfrage. Sie lässt sich sehr zuverlässig mit orthopädisch-traumatologischem Fachwissen beantworten. Für Bewertungen durch den Sachverständigen lässt sie keinen Raum. Das ist anders bei der Frage: „Erlitt der Kläger durch den Unfall einen Oberschenkelbruch?“ Sie ist deutlich komplexer und ohne Wertungen kaum zu beantworten.

Durch eine unklare Formulierung des Gutachtenauftrags gibt der Richter also einen Teil seiner Entscheidungsmacht im Prozess an den Gutachter ab. Trotzdem sollte man sich keinen Illusionen hingeben. Reine Sachfragen, die den Gutachter auf die kognitive-rationale Analyse beschränken, sind selten ausreichend. Hilfreich für die

183 H. Kater, Gutachten (Fn. 24), S. 54.

184 BGH NJW 2005, S. 2859.

185 A. Nack, Abhängigkeit des Richters vom Sachverständigen, GA 2009, S. 201 (206), vertritt auf der Grundlage seiner praktischen Erfahrung als BGH-Richter die Ansicht, dass dies zu wenig geschehe.

186 H. Brettel/H. Vogt, Begutachtung (Fn. 30), S. 99.

187 Instruktiv zum Unterschied zwischen Sach-und Bewertungsfragen R. Hepp, Sach- und Bewertungsfragen an den medizinischen Sachverständigen im Rechtsstreit, NZV 2013, S. 581.

188 R. Hepp, Bewertungsfragen (Fn. 188), S. 581. 
Entscheidung des Gerichts werden in der Regel erst Antworten auf komplexe Fragen. Komplexere Fragen aber sind ohne Wertungen kaum zu beantworten. ${ }^{189}$

\section{Interdiszipinäre Kommunikationskompetenz}

Der Richter ist kein Vollzugsgehilfe des Sachverständigen. ${ }^{190}$ Er darf schon aus rechtlichen Gründen dem vorgelegten Gutachten nicht blind folgen. Der Richter muss sich mühsam in die fremde Materie und in das fremde Denken einarbeiten. ${ }^{191} \mathrm{Er}$ muss versuchen, die Ausführungen des Sachverständigen so weit es irgend geht zu verstehen, einzuordnen und zu würdigen. Das wird nur selten durch die reine Lektüre des Gutachtens möglich sein. Deshalb ist in der Regel ein ausführliches Gespräch zwischen Richtern und Sachverständigen über das vorgelegte Gutachten in der mündlichen Verhandlung nötig. ${ }^{192}$ Richter müssen im intensiven Gespräch mit dem Gutachter während der Hauptverhandlung alle Fachfragen klären. ${ }^{193}$ Dann sind Sie - soweit das überhaupt möglich ist ${ }^{194}$ - in der Lage, ein sachlich fundiertes Urteil zu sprechen. Wie wichtig das Gespräch mit dem Gutachter in der mündlichen Verhandlung ist, unterstreicht die Rechtsprechung des BGH. Er sieht das rechtliche Gehör einer Partei verletzt, wenn ihrem Antrag auf Befragung des Gutachters nicht entsprochen wird. ${ }^{195}$

Weil es zwangsläufig interdisziplinär geführt werden muss, ist dieses Gespräch schwierig. Schon allein die (Fach)Sprachbarriere zu überwinden, ist nicht leicht. ${ }^{196}$ Fachbegriffe einzelner Wissenschaften lassen sich nicht einfach in juristische Kategorien übersetzen. ${ }^{197}$ Die interdisziplinäre Kommunikation zwischen Gericht und

189 Um beim oben geschilderten Beispiel zu bleiben: ob ein Kläger nach einem Unfall einen Oberschenkelbruch erlitten hat, ist für einen Prozess in der Regel wenig relevant. Entscheidend ist die komplexere Frage, ob der Oberschenkelbruch durch den Unfall verursacht wurde.

190 G. Wannagat, Sachverständige (Fn. 47), S. 349 (350).

191 H. Kater, Gutachten (Fn. 24), S. 48 ff. am Beispiel der Medizin. Das gilt aber auch für Gutachten aus anderen Wissenschaftsgebieten.

192 So auch G. Wannagat, Sachverständige (Fn. 47), S. 349 (351) auf der Basis jahrzehntelanger Erfahrungen in der Sozialgerichtsbarkeit. Ähnlich H. Franzki, Diener (Fn. 4), S. 314 (319), der die Bedeutung der Erläuterung des Gutachtens in der mündlichen Verhandlung unterstreicht. Anderer Meinung ist M. Dölp, Der Sachverständige im Strafprozess, ZRP 2004, S. 235 (236), der den Sachverständigen aus der strafrechtlichen Hauptverhandlung heraushalten will, um das Verfahren effektiver und kostengünstiger zu machen.

193 Instruktiv dazu die empirische Studie von C.O. Schmidt/O.B. Scholz (Fn. 4), S. 414. Danach übernimmt ein Richter bei der Schuldfähigkeitsbegutachtung im Strafprozess fast ausnahmslos das Ergebnis des mündlichen Gutachtens. Beim schriftlichen Gutachten ist die Übernahmequote geringer. Das belegt die Bedeutung des direkten Gesprächs zwischen Richter und Gutachter in der Verhandlung.

194 Zur allgemeinen Erkenntnisskepsis, die davon ausgeht, dass Erkenntnis grundsätzlich nicht möglich ist, ganz pragmatisch H. Kater, Gutachten (Fn. 24), S. 50.

195 BGH Beschluss vom 15.3.2006, IV ZR 182/05, Rn. 6.

196 Zur Sprachbarriere H. Franzki, Diener (Fn. 4), S. 314 (319), und H. Brettel/H. Vogt, Begutachtung (Fn. 30), S. 100.

197 K. Detter, Der Sachverständige im Strafverfahren - eine Bestandsaufnahme - NStZ 1998, S. 57 (60) $\mathrm{m}$. Beispielen. 
Gutachter wird immer dann besonders schwierig, wenn und weil der Gutachter das Weltbild des Gerichts infrage stellt. ${ }^{198}$ Gerade bei psychiatrischen Gutachten passiert das nicht selten. Das hat eine grundsätzliche Ursache. Psychiatern geht es um das Verstehen, Richtern geht es um das Urteilen. ${ }^{199}$ Kommunikationsschwierigkeiten und Konflikte sind damit programmiert.

Beim interdisziplinären Gespräch zwischen Gericht und Gutachter geht es nicht nur um fachspezifische Begriffsbildungen und spezielle Definitionen. Andere Wissenschaftsbereiche haben andere Kommunikations-und Ausdrucksmittel als die Juristen. Ingenieure als Sachverständige etwa arbeiten auch mit mathematischen Formeln, Diagrammen, Bildern und Zeichnungen. ${ }^{200}$ Sich darauf einzulassen ist für Richter oft schwierig. Denn ihr bevorzugtes Kommunikationsmittel sind - geschriebene und gesprochene - Worte. ${ }^{201}$

Selbst wenn das gelingt, müssen beide Seiten unterschiedliche Denkweisen, Wissenshintergründe, Erkenntnismethoden und Argumentationsmuster verarbeiten können. Das erfordert eine grundsätzliche Offenheit für das andere Fach, nicht unbeträchtliche intellektuelle Kompetenzen und nicht zuletzt geschulte Kommunikationsfähigkeiten - sowohl bei den Richtern als auch bei den Sachverständigen.

Das lässt sich am Beispiel medizinischer Sachverständiger vor Gericht illustrieren. Mediziner sind Erfahrungswissenschaftler und wollen biologische Zusammenhänge erkennen. Diese Zusammenhänge sind selbst in gut erforschten Bereichen noch lange nicht vollständig verstanden. Gleichzeitig ist die Schwankungsbreite hoch. Es gibt kaum den einen typischen Fall. Abweichungen, Ausnahmen, Modifikationen sind fast die Regel. Das hat eine methodische Konsequenz: In der Medizin gibt es kaum sichere Wahrheiten; mehr oder weniger häufig bestätigte Hypothesen dominieren. Sichere Aussagen sind selten möglich. Mediziner arbeiten deshalb nicht mit Gewissheiten und sicheren Aussagen, sondern mit Wahrscheinlichkeiten. ${ }^{202}$ Das irritiert Juristen oft. Sie denken deduktiv im Sinne der klassischen Logik. Dabei kommen sie mit Hilfe streng logischer Denkoperationen zu eindeutigen Folgerungen. Wahrscheinlichkeiten und stochastische Überlegungen sind ihnen eher fremd. Besonders heftig treffen beide Denkwelten aufeinander, wenn es um die Kausalität in Arzthaftungsprozessen geht. Gibt es eine Kausalität, oder gibt es keine Kausalität? Das ist

198 N. Nedopil, Verständnisschwierigkeiten zwischen dem Juristen und dem psychiatrischen Sachverständigen, NStZ 1999, S. 433 (434 f.).

199 N. Nedopil, Verständnisschwierigkeiten zwischen dem Juristen und dem psychiatrischen Sachverständigen, NStZ 1999, S. 433 (435).

200 B. Voth, 30 Jahre Gerichtsgutachter - ein Erfahrungsbericht, BauR 1988, S. 666 (674).

201 Grundsätzlich dazu V. Boehme-Neßler, Bilderrecht, Berlin 2010, S. 115.

202 R. Jungbecker, Unterschiede zwischen medizinischem und juristischem Denken - ein bequemes Alibi?, in: Arbeitsgemeinschaft Rechtsanwälte im Medizinrecht (Hrsg.), Der medizinische Sachverständige, Köln 1995, S. 17. 
die Frage, die Gerichte beantwortet haben wollen. Für Mediziner gibt es aber nur sehr wenige sichere Kausalitäten. ${ }^{203}$ Dafür sind die Vorgänge im menschlichen Körper einfach zu komplex und noch zu wenig verstanden. Ein anderes Beispiel: Die Schuld hat eine zentrale Bedeutung im Strafrecht und im Strafprozess. In den psychologisch-psychiatrischen Kategorien gibt es den Begriff der Schuld aber nicht. ${ }^{204}$ Eine Diskussion über Schuldfähigkeit und Verantwortung zwischen Psychiatern und Juristen ist schon deshalb schwierig.

Richter sind in foro oft mit dem Grundproblem der Interdisziplinarität konfrontiert: Wer keine Kenntnisse des anderen Fachgebiets hat, findet keine Ansatzpunkte für Fragen oder Kritik und weiß nicht wirklich, wo die relevanten Probleme liegen. Kurz: Wer als Richter nichts über ein Fachgebiet weiß, kann dem Gutachter nicht die richtigen Fragen stellen. ${ }^{205}$

Ein einfacher Weg aus diesen Schwierigkeiten bietet sich an: Die Richter drängen die Experten, juristische Bewertungen abzugeben. ${ }^{206}$ Selbstverständlich ist das rechtswidrig. Denn die Richter verweigern sich ihrer ureigenen Aufgabe. Und die Gutachter, überschreiten ihre Kompetenzen zu. ${ }^{207}$ Genau so einfach und rechtswidrig ist ein zweiter Weg. Angesichts der hohen Arbeitsbelastung im Richteralltag liegt es deshalb nahe, dem Gutachter „blind“ zu vertrauen - und dadurch viel Arbeit und Zeit zu sparen. ${ }^{208}$

Der interdisziplinäre Diskurs zwischen Gericht und Gutachter ist gleichzeitig besonders schwierig und völlig unverzichtbar. Letztlich müsste diese Erkenntnis eine rechtspolitische Auswirkung haben: Spezifische Kommunikationskompetenzen, die zum interdisziplinären Diskurs befähigen, müssten in die Ausbildungen sowohl der Richter als auch der Sachverständigen aufgenommen werden.

\section{Professionelle Plausibilitätskontrolle und fundiertes Vertrauen}

Wie kann ein Richter ohne besondere Fachkenntnisse ein Sachverständigengutachten würdigen und in seiner Entscheidung verarbeiten? Der Königsweg ist die Plausibilitätskontrolle. Aber selbst eine professionelle Plausibilitätskontrolle versetzt den Richtern nicht in die Lage, das Sachverständigengutachten fachlich bis ins letzte zu

203 F.-J. Schulte, Diskussionsbeitrag zu R. Jungbecker, in: Arbeitsgemeinschaft Rechtsanwälte im Medizinrecht (Hrsg.), Der medizinische Sachverständige, Köln 1995, S. 20 (21 f.).

204 N. Konrad/W. Rasch, Psychiatrie (Fn. 42), S. 20.

205 K. Oehler, Zur Problematik der Sachverständigenauswahl, ZRP 1999, S. 285 (286).

206 K. Oehler, Problematik (Fn. 207), S. 285 (287) bezeichnet das als „Kompetenzparasitismus“. Dazu aus der Sicht eines forensischen Psychiaters N. Nedopil, Verständnisschwierigkeiten zwischen dem Juristen und dem psychiatrischen Sachverständigen, NStZ 1999, S. 433 (434).

207 Ausführlich zu den Kompetenzgrenzen der Psychogutachter K. Foerster, Der psychiatrische Sachverständige zwischen Norm und Empirie, NJW 1983, S. 2049 (2051).

208 Instruktiv dazu die Episode, die N. Nedopil, Verständnisschwierigkeiten zwischen dem Juristen und dem psychiatrischen Sachverständigen, NStZ 1999, S. 433 (434), schildert. 
durchdringen. Am Ende muss er dem Sachverständigen letztlich bis zu einem gewissen Grad Vertrauen. Das darf allerdings kein blindes Vertrauen sein.

\section{Plausibilitätskontrolle}

Im Rahmen der Beweiswürdigung muss der Richter das Sachverständigengutachten einschätzen und werten. ${ }^{209}$ Das ist natürlich schwierig. Denn er hat den Sachverständigen beauftragt, weil er kein eigenes Fachwissen hat. Das wäre aber nötig, um das Gutachten in allen Details würdigen zu können. Allerdings ist diese Situation nicht ungewöhnlich: Letztlich müssen alle Menschen immer wieder Sachverhalte und Informationen einschätzen, die sie letztlich nicht wirklich beurteilen können. Das Mittel der Wahl dabei ist die Plausibilitätskontrolle. Das ist auch die Methode, die ein Gericht bei der Würdigung eines Gutachtens anwendet. ${ }^{210}$ Dabei lassen sich zwei Dimensionen unterscheiden: eine formale und eine materielle. ${ }^{211}$

Die formelle und indirekte Plausibilitätskontrolle ist relativ einfach. Der Richter prüft die Sachkunde, die Unabhängigkeit und die Neutralität des Gutachters. Er informiert sich über die Reputation, die der Gutachter in der Fachwelt genießt. Teilweise findet das schon vor der Bestellung des Sachverständigen statt. Aber auch, wenn das Gutachten vorliegt, ist die formale Plausibilitätskontrolle noch nicht beendet. Aus den verwendeten wissenschaftlichen Quellen, dem Überblick über den Forschungsstand und aus einzelnen Formulierungen lassen sich in einer Gesamtschau Rückschlüsse auf die Fachkunde, die Neutralität, die Objektivität und die Unabhängigkeit des Gutachters ziehen. Zur indirekten Plausibilitätskontrolle gehört auch die Verfahrenskontrolle. ${ }^{212}$ Der Wert des Sachverständigengutachtens hängt auch davon ab, ob der Gutachter die Grundsätze eines fairen Verfahrens beachtet hat.

Die materielle, indirekte Plausibilitätskontrolle ist ungleich schwieriger. Trotzdem muss das Gericht sie durchführen. Der Richter muss das Gutachten nach Kräften auf seine logische und wissenschaftliche Begründung nachprüfen. ${ }^{213} \mathrm{Er}$ muss versuchen, die Gedankenführung des Gutachtens nachzuvollziehen. Letztlich geht es um die Schlüssigkeit der Begründungen, die logische Geschlossenheit der einzelnen Schlussfolgerungen und die Plausibilität des gefundenen Ergebnisses. ${ }^{214}$ Das wird naturgemäß nur begrenzt möglich sein. Die fehlende echte Sachkunde des Richters setzt hier Grenzen.

210 Dazu schon BGHSt 8, 113 (118).

211 Ähnlich F. Nicklisch, Generalbericht (Fn. 154), S. 252 f.

212 Dazu F. Nicklisch, Generalbericht (Fn. 154), S. 253 f.

213 BVerwGE 17, 342 (343).

214 F. Nicklisch, Generalbericht (Fn. 154), S. 254. 


\section{Fundiertes, kein blindes Vertrauen}

So kompetent ein Richter auch sein mag: Die Fachkompetenz des Gutachters und die fachliche Qualität des Gutachtens wird er nicht bis ins letzte beurteilen können. Früher oder später kommt er an den Punkt, an dem er dem Sachverständigen vertrauen muss. ${ }^{215}$ Das bedeutet: Jede Beweiswürdigung eines Sachverständigengutachtens ist mit einem Restrisiko verbunden, das unvermeidlich ist. Nach einer professionellen Plausibilitätskontrolle dürfte das Risiko aber überschaubar sein. Ohne ein Minimum an Vertrauen wird der Richter aber keine Entscheidung treffen können.

Vertrauen hat eine große Bedeutung für das Handeln in sozialen Situationen. Wer vertraut, kann auch in Situationen handeln, deren Komplexität er nicht vollständig durchschaut. ${ }^{216}$ Vertrauen ermöglicht es, kalkuliert Risiken einzugehen und dadurch die eigenen Handlungsoptionen zu erweitern. Es ist eine bewährte Methode, mit den Folgen von Undurchsichtigkeit, Unwissen und sozialer Kontingenz umzugehen. ${ }^{217}$ Wenn der Richter nach einer professionellen Plausibilitätskontrolle dem Gutachter vertraut, kann er auch hochkomplexe Fälle entscheiden, deren fachliche Implikationen er selber nicht völlig durchschaut.

\section{G. Richter und Gutachter - Ein ewiger Balanceakt}

Die Macht der Gutachter ist also groß. Aber sie ist nicht unbegrenzt. Zu einem Teil ist sie strukturell begründet. Zu einem anderen, großen Teil ist sie aber nur deshalb so groß, weil Gerichte sie ihnen einräumen. Sie ist oft nur „geliehen“. Die Arbeit von Richtern und Sachverständigen lässt sich - anders als die rechtliche Theorie es vorsieht - nicht trennscharf abgrenzen. Das hat strukturelle Gründe, die sich nicht verändern lassen. Gleichzeitig sind Ziele und Denkweisen der Justiz nicht dieselben wie die der Gutachter. ${ }^{218}$ Für die Machtfrage im Verhältnis von Richter und Sachverständigen gibt es deshalb keine eindeutige, statische Lösung. Beide müssen zusammenarbeiten, um ein optimales Urteil zu erzielen. ${ }^{219}$ Wie die Machtbalance zwischen beiden aussieht, muss jedes Mal von neuem im Einzelfall austariert werden. Der Richter muss sich immer dessen bewusst sein, dass er das Urteil spricht und dafür verantwortlich ist. Er muss das Know-how des Sachverständigen nutzen, darf sich aber nicht hinter ihm verstecken. Genauso muss er darauf achten, dass er den Gut-

215 Instruktiv dazu BGHSt 7, 239.

216 G. Simmel, Soziologie. Untersuchungen über die Formen der Vergesellschaftung, 5. Auflage Berlin 1968, S. 263, bezeichnet Vertrauen unter diesem Aspekt als mittleren Zustand zwischen Wissen und Nichtwissen.

217 So ganz prägnant C. Offe, Wie können wir unseren Mitbürgern vertrauen? in: M. Hartmann/C. Offe (Hrsg.), Vertrauen - die Grundlage des sozialen Zusammenhalts, Frankfurt a.M. 2001, S. 250.

218 Dazu am Beispiel der Psychiater N. Nedopil, Verständnisschwierigkeiten zwischen dem Juristen und dem psychiatrischen Sachverständigen, NStZ 1999, S. 433 (438f.).

219 H. Pieper u.a., Sachverständige im Zivilprozeß, 1982, S. 87 ff. sehen das Verhältnis zwischen Richter und Sachverständigem denn auch als „Arbeitsgemeinschaft“. 
achter nicht unter Erwartungsdruck setzt und ihm das gewünschte Ergebnis suggeriert. ${ }^{220}$ Er muss offen für das Ergebnis des Gutachtens sein - und das dem Gutachter explizit und implizit kommunizieren. Der Sachverständige soll seine Expertise in den Prozess einbringen. Er soll ein unabhängiger und objektiver wissenschaftlicher Ratgeber sein. Er ist kein Richter. ${ }^{221}$ Wenn sich beide ihrer unterschiedlichen, aber unverzichtbaren Rolle bewusst sind, sollte die Zusammenarbeit mit der nötigen Souveränität fruchtbar gelingen können. Im Idealfall führt der nötige Balanceakt zu einem Urteil, das vom Gericht gesprochen und verantwortet wird - und gleichzeitig die Sachkunde des Gutachters verarbeitet hat.

220 So auch K. Detter, Der Sachverständige im Strafverfahren - eine Bestandsaufnahme, NStZ 1998, S. 57.

221 Eindrücklich und prägnant zur Rolle des Sachverständigen N. Nedopil/J. L. Müller, Psychiatrie (Fn. 2), S. 32 f. m.w.N. 